\title{
Virus-induced cochlear inflammation in newborn mice alters auditory function
}

\author{
Cathy Yea Won Sung, ${ }^{1}$ Maria C. Seleme, ${ }^{2}$ Shelby Payne, ${ }^{3}$ Stipan Jonjic, ${ }^{4}$ Keiko Hirose, ${ }^{3}$ \\ and William Britt $1,2,5$ \\ 1Department of Microbiology and 2Department of Pediatrics, University of Alabama School of Medicine, Birmingham, \\ Alabama, USA. ${ }^{3}$ Department of Otolaryngology, Washington University, St. Louis, Missouri, USA. ${ }^{4}$ Department of \\ Histology and Embryology, Faculty of Medicine, University of Rijeka, Rijeka, Croatia. ${ }^{5}$ Department of Neurobiology, \\ University of Alabama School of Medicine, Birmingham, Alabama, USA.
}

\begin{abstract}
Although human cytomegalovirus (HCMV) is a known cause of sensorineural hearing loss in infants with congenital HCMV (cCMV) infections, mechanisms that contribute to sensorineural hearing loss (SNHL) in infants with CCMV infection are not well defined. Using a murine model of CMV infection during auditory development, we have shown that peripheral infection of newborn mice with murine CMV (MCMV) results in focal infection of the cochlea and virus-induced cochlear inflammation. Approximately $50 \%-60 \%$ of infected mice exhibited increased auditory brainstem response (ABR) thresholds across a range of sound frequencies. Histological analyses of the cochlea in MCMV-infected mice with elevated ABR thresholds revealed preservation of hair cell (HC) number and morphology in the organ of Corti. In contrast, the number of spiral ganglion neurons (SGN), synapses, and neurites connecting the cochlear HC and SGN nerve terminals were decreased. Decreasing cochlear inflammation by corticosteroid treatment of MCMV-infected mice resulted in preservation of SCN and improved auditory function. These findings show that virus-induced cochlear inflammation during early auditory development, rather than direct virus-mediated damage, could contribute to histopathology in the cochlea and altered auditory function without significant loss of HCs in the sensory epithelium.
\end{abstract}

Conflict of interest: The authors have declared that no conflict of interest exists.

Copyright: (c) 2019 American Society for Clinical Investigation

Submitted: March 21, 2019

Accepted: August 1, 2019

Published: August 8, 2019.

Reference information: /Cl Insight. 2019;4(17):e128878. https://doi. org/10.1172/ji.insight.128878

\section{Introduction}

Human cytomegalovirus (HCMV) infection is the most common congenital viral infection in humans, with approximately 20,000 infants with congenital HCMV (cCMV) infections born every year in the United States $(1,2)$. Of these, about 3,000 infants with cCMV infection will develop long-term neurological damage, including sensorineural hearing loss (SNHL) in approximately $12 \%$ of infected infants (3-6). The degree of hearing loss (HL) in infected infants is highly variable, ranging from moderate to profound, and can be unilateral or bilateral $(7,8)$. In addition, HCMV-infected infants can experience late-onset HL months to years after birth $(1,7)$. Although antiviral drugs are used to treat infants with severe cCMV infection, their toxicity has restricted their use in pregnant women due to potentially teratogenic effects in the fetus (9). Studies of the inner ears (cochlea) of terminated human fetuses infected with HCMV have described virus-infected cells in cochlear structures, including the stria vascularis, spiral ganglion neurons (SGN), supporting cells (SCs), and Reissner's membrane $(10,11)$. Abundant mononuclear cell infiltrates were also noted $(10,11)$. Other congenital virus infections such as rubella are believed to cause SNHL through a mechanism of direct viral damage to the cochlea, resulting in cell death in the organ of Corti and damage to the stria vascularis $(12,13)$.

To study the pathogenesis of SNHL in CCMV infection, we have developed a mouse model in which newborn mice are infected i.p. with nonlethal doses of murine CMV (MCMV), a virus with high genetic relatedness, similar replication program, and commonalities in biologic behavior with HCMV (14). Following i.p. injection of MCMV, the virus spreads hematogenously to the CNS (including the cochlea). This model relies on the observation that, developmentally, the auditory system of the newborn mouse is similar to that of an early second trimester human fetus. The auditory system in mice develops postnatally, and although the onset of hearing is around P12-P14, the auditory system fully matures around P20 (15-18). Thus, this model has allowed investigation of the impact of virus infection during the development of the auditory system. 
We previously demonstrated that MCMV infection in newborn mice resulted in a moderate to severe increase in auditory brainstem response (ABR) thresholds in approximately $40 \%-50 \%$ of infected mice (19). Increasing the inoculum size increased the incidence of animals exhibiting elevated thresholds and resulted in higher incidence of mice with more severe ABR abnormalities (19). In addition, about $30 \%$ of infected mice exhibited progressive increases in ABR thresholds within the first 3 months of life (19). Thus, phenotypes of HL in MCMV-infected mice recapitulated several of the most salient clinical features of HL that follow cCMV infection in infants. Our previous findings in this model indicated that MCMV infection during auditory development resulted in HL in infected mice that could not be correlated with the amount of virus in the cochlea but, instead, was associated with the expression of virus-induced proinflammatory cytokines and chemokines in the cochlea (19). However, in this earlier study, we did not comprehensively describe the impact of MCMV infection on the morphology of cochlear structures that could contribute to elevated ABR thresholds. In the current study, we have cataloged the histopathological changes within the cochlea of MCMV-infected animals and identified multiple lesions that could contribute to HL in MCMV-infected mice. Surprisingly, we found no evidence of hair cell (HC) loss in mice with elevated ABR thresholds, arguing that the mechanism of $\mathrm{HC}$ dysfunction in this model remains to be defined. In addition, we directly tested the hypothesis that virus-induced inflammation, and not lytic virus replication, resulted in the observed histopathological and functional abnormalities in the mature cochlea of mice infected in the newborn period by treating infected mice with corticosteroids. Our results were consistent with virus-induced inflammation as a mechanism of SNHL and histopathological findings in the cochlea of mice infected with MCMV during auditory development. These findings raised the possibility that similar mechanisms could contribute to SNHL associated with cCMV infections in infants.

\section{Results}

$M C M V$ replicates in the cochlea of newborn mice and induces robust inflammatory responses. To define the kinetics of cochlear infection with MCMV, newborn mice (P0) were injected i.p. with 500 PFU MCMV, and MCMV DNA was quantified in cochlea from P4-P44. In contrast to our previous studies, we utilized a molecular clone of the Smith strain of MCMV (MCMV-repaired) that represented a homogenous population of virus $(14,19)$. MCMV DNA was initially detected on P4 and reached peak levels by P12, with viral DNA declining throughout the postnatal period (Figure 1A). MCMV infection was focal in the cochlea, with small numbers of MCMV-infected cells present in foci that were, in turn, widely scattered throughout the cochlea (1-2 foci/section) (Figure 1B) $(14,20)$. MCMV-infected cells were detected in SGNs in the modiolus and the stria vascularis (Figure 1B) (19). However, analysis of multiple sections from a large number of cochleae failed to reveal any evidence of virus infection in resident cells of the organ of Corti (Figure 1B). Together, these data indicate that, following peripheral infection in newborn mice, the virus spread hematogenously to the cochlea and established foci of infection in several regions of the cochlea, but importantly, it spared cells of the sensory epithelium in the cochlea.

To define the kinetics of virus-induced inflammation in the cochlea, we quantified expression of an interferon-stimulated gene (ISG), IFIT1, and a proinflammatory cytokine, TNF- $\alpha$, in the cochlea of infected mice between P4-P44. Consistent with the increase in viral DNA during the first 12 days after infection, the expression of IFIT1 increased significantly and then decreased as virus was cleared from the cochlea (Figure 1C). In contrast, expression of TNF- $\alpha$ in cochlea peaked slightly later and decreased more slowly than the expression of IFIT1 (Figure 1D). In addition, mononuclear cells expressing Iba-1, a marker for activated cells of the myeloid lineage, were increased in P8 cochlea in infected mice as compared with controls (Figure 1B). Of note, in some sections, Iba- $1^{+}$mononuclear cells accumulated near foci of MCMV-infected cells (Figure 1B). Together, these results indicated that, following MCMV dissemination and establishment of a focal infection in the inner ear, a robust inflammatory response characterized by the expression of ISGs, proinflammatory cytokines, and activated myeloid cells could be detected in the cochlea early after infection.

$M C M V$ infection in newborn mice is associated with altered auditory function in young adult mice in the absence of $H C$ loss. To confirm that cochlear infection in newborn mice resulted in altered auditory function, we utilized ABR testing to quantify evoked potentials in the mouse cochlea and brain in response to auditory stimuli in noninfected control mice and in mice infected as newborns with either 200 or 500 PFU of MCMV. Hearing was measured at P32, a time point that virtually assured that the auditory system would be fully mature in all 
A

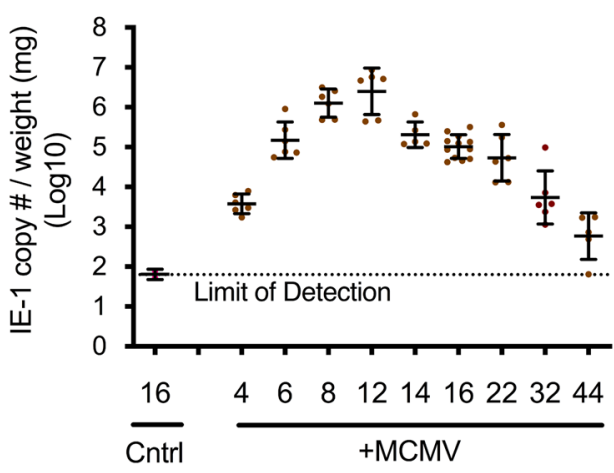

Postnatal day
B

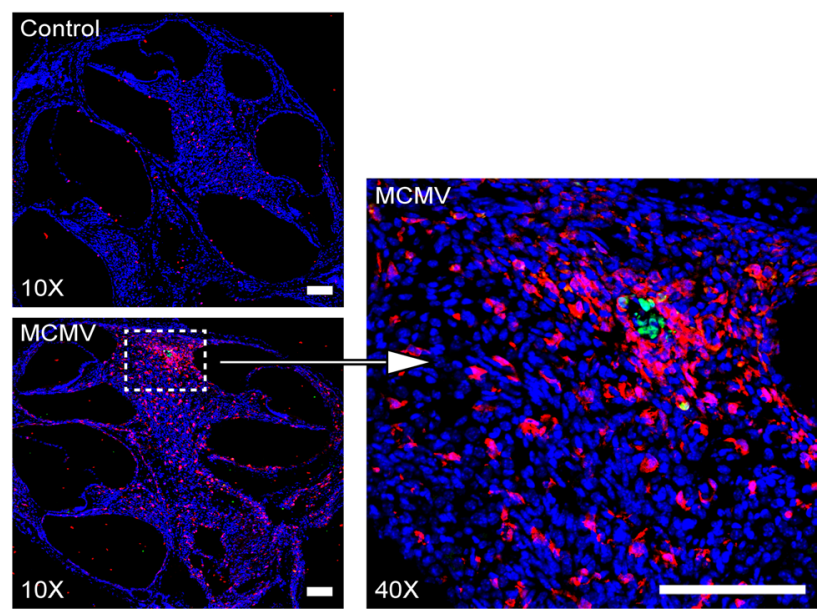

D

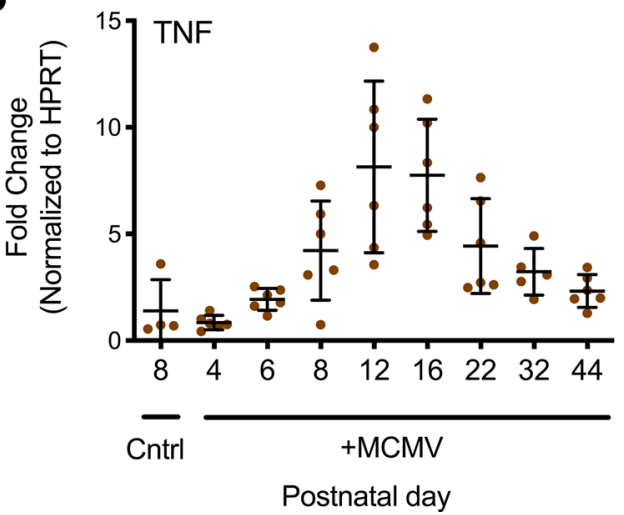

Figure 1. I.p. inoculation of newborn mice with MCMV results in cochlear infection and inflammation. (A) MCMV DNA was extracted from the cochlea and quantified as described in Methods, with each data point representing genome copy number in single cochlea. (B) Representative images of cochleae from noninfected, control (top) and MCMV-infected (bottom) mice displaying inflammatory response. Midmodiolar cochlear sections of P8 MCMV-infected mice were immunostained for MCMV IE-1 protein (green) and Iba-1 (red), a marker for activated myeloid cells, and nuclear stain (blue) (10× magnification). A higher-magnification of area of interest (boxed area) in cochlea from MCMV-infected mouse is shown on the right (40x magnification). A total of $n=3$ mice $/ n=6$ cochleae were stained. Scale bars: $100 \mu \mathrm{m}$. (C) Expression of interferon-stimulating gene (ISG), IFIT1, and (D) proinflammatory cytokine TNF- $\alpha$ were measured by reverse transcription PCR at various time points from RNA extracted from cochlea as described in Methods. HPRT was used as internal control, and fold change was normalized to the noninfected, control cochlea. For each time point, $n=4-6$ mice were assayed.

mice (15-17). Auditory stimuli were provided to mice in the form of broadband click stimuli (Figure 2A) and frequency-dependent pure tones ranging from $4-48 \mathrm{kHz}$ (Figure 2B) to measure hearing thresholds as defined by the lowest intensity stimulus ( $\mathrm{dB}$ ) at which ABR waveforms could be detected. The median of ABR thresholds in the group of MCMV-infected animals, measured by both broadband click and pure tone, were significantly elevated compared with a noninfected, control group of animals (Figure 2, A and B). Ears from MCMV-infected animals showed significant increases in the ABR threshold at all frequencies when compared with noninfected controls, suggesting that impairment in auditory function was generalized and not limited to a specific region in the cochlea (Figure 2B). We also assessed the function of outer HCs (OHC) using distortion product otoacoustic emission (DPOAE) testing (Figure 2C). Mice with elevated ABR thresholds measured by broadband click exhibited increased DPOAE thresholds at all frequencies, thus providing evidence of $\mathrm{OHC}$ dysfunction in infected mice (Figure 2C). Lastly, we quantified the amplitude and latency of wave I of the ABR as measures of the conduction of the output from the inner HCs (IHC) in the cochlea (Figure 2D). MCMV-infected animals with ABR click thresholds between 60 and $90 \mathrm{~dB}$, indicative of severe $\mathrm{HL}$, displayed impairment in the amplitude of $\mathrm{ABR}$ wave I growth measured at 8 and $32 \mathrm{kHz}$ frequencies, while infected animals with normal $\mathrm{ABR}$ thresholds displayed comparable amplitude of ABR wave I growth compared with the noninfected, control animals (Figure 3 , A and B). Furthermore, the latency of wave I was delayed in infected mice with significant elevations of ABR thresholds, whereas there were no significant delays in latency in infected animals with normal ABR thresholds 
A

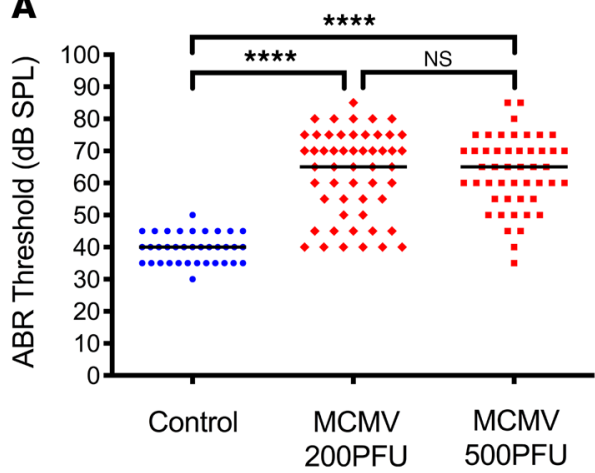

C

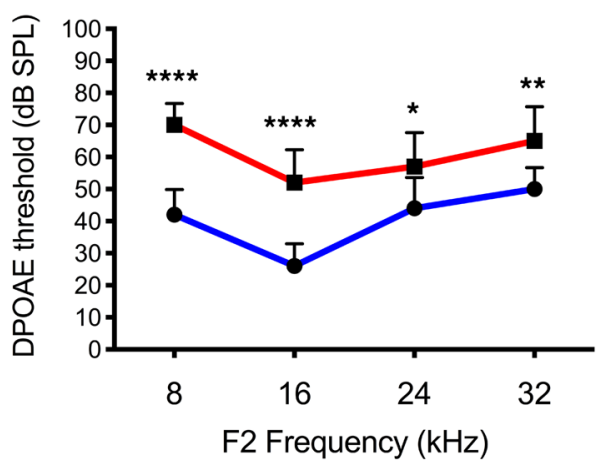

B

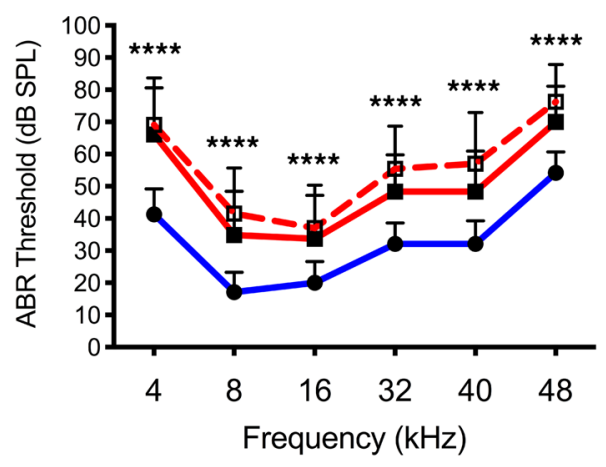

D

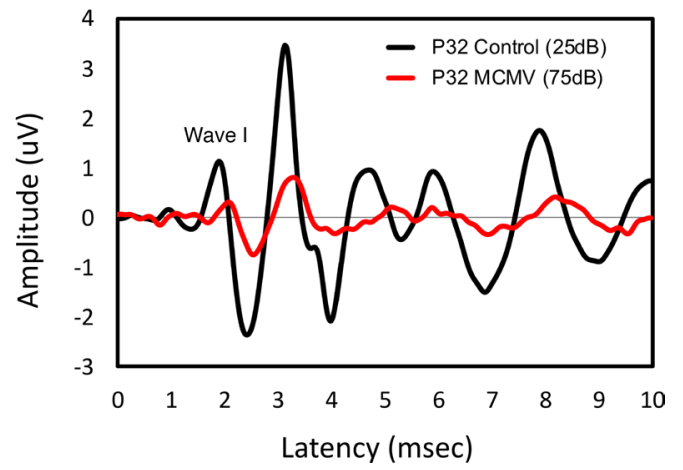

Figure 2. MCMV infection of newborn mice results in moderate to severe hearing loss. (A-C) ABR thresholds determined at P32 in noninfected control mice and mice infected as newborns with 200 or 500 PFU of MCMV. (A) Broadband clicks were presented to the animals in the following groups: noninfected, $n=18$ mice/36 ears; 200 PFU MCMV $n=27$ mice/54 ears; 500 PFU MCMV $n=24$ mice/48 ears. $P$ values calculated with 1-way ANOVA (Kruskal-Wallis test) with Dunn's comparisons test. (B) Frequency-dependent tone-pips were used to determine the ABR thresholds in control and infected mice. Data shown as mean \pm SD; noninfected control mice (blue solid line), $n=12$ mice/24 ears; 200 PFU (red dashed line) MCMV, $n=27$ mice/54 ears; and 500 PFU (red solid line) MCMV, $n=12$ mice/24 ears. (C) For DPOAE testing, noninfected control mice (blue solid line), $n=8$ mice/16 ears, and infected mice with elevated ABR click thresholds above $60 \mathrm{~dB}$ (red solid line), $n=7 / 14$ ears were used. $P$ values for frequency tone-pip and DPOAE were determined by 2-tailed unpaired $t$ test (Mann-Whitney $U$ test). (D) Sample ABR tracings from noninfected, control mouse (black) and MCMV-infected mouse (red) with $A B R$ threshold of $25 \mathrm{~dB}$ and $75 \mathrm{~dB}$, respectively, measured with broadband clicks. Note designation of wave I in control tracing with amplitude expressed in $\mu \mathrm{V}$ and latency in msec. ${ }^{*} P<0.05 ;{ }^{* *} P<0.01 ;{ }^{* * *} P<0.0001$.

(Figure 3, C and D). Collectively, these results suggested that MCMV infection in some but not all newborn mice led to moderate to severe loss of normal auditory functions, as measured by ABR and DPOAE testing, and alterations in the amplitude and latency of ABR wave I that, when viewed together, represent the summated response from SGNs and cranial nerve VIII.

The number of HCs and SC subtypes, specifically Deiters' cells, in MCMV-infected and control mice on P32 were compared to determine if increased thresholds detected in ABR and DPOAE testing in MCMV-infected mice was accompanied by damage or loss of cells in the sensory epithelium. For these experiments, cochleae from mice with ABR thresholds above $60 \mathrm{~dB}$ were compared with cochleae from noninfected, control animals. Immunological identification of HCs (MyosinVIIa) and Deiters' cells (Sox2), nonsensory cells located adjacent to OHC, revealed comparable numbers of IHC, OHC, and Deiters' cells in the cochlea of noninfected (ABR threshold of $30 \mathrm{~dB}$ ) and MCMV-infected mice (ABR threshold of $75 \mathrm{~dB}$ ) (Figure 4). This indicated that the elevation of ABR and DPOAE thresholds in MCMV-infected mice were not associated with loss of HC or Deiters' cells or with recognizable morphological changes at the level of conventional confocal microscopy in these cell types when compared with similar preparations from noninfected, control mice with normal ABR thresholds. Thus, in this model of virus-induced HL, elevation of ABR thresholds secondary to $\mathrm{HC}$ dysfunction is present in the absence of overt loss or damage of the sensory epithelium.

$M C M V$-infected mice display reduced numbers of inner HC synapses and neurite density. To further define the impact of MCMV infection and virus-induced inflammation during early cochlear development, we quantified the number of synapses between HCs and the SGN nerve terminals. The organ of Corti from 
A
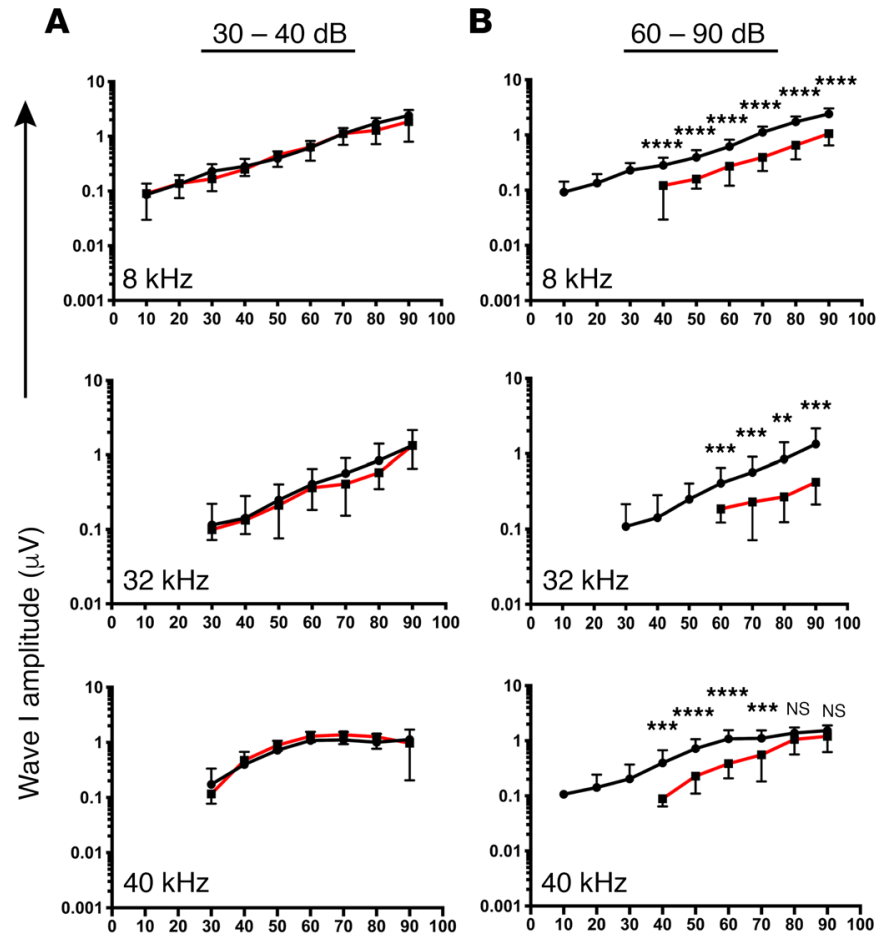

C
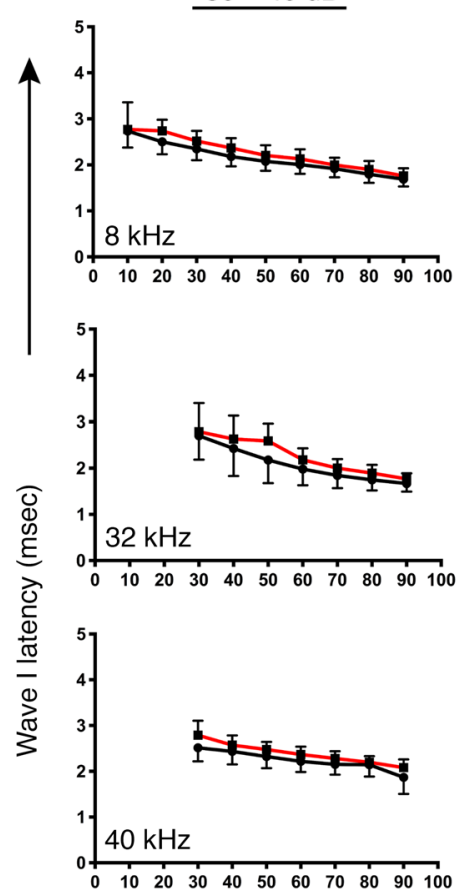

D
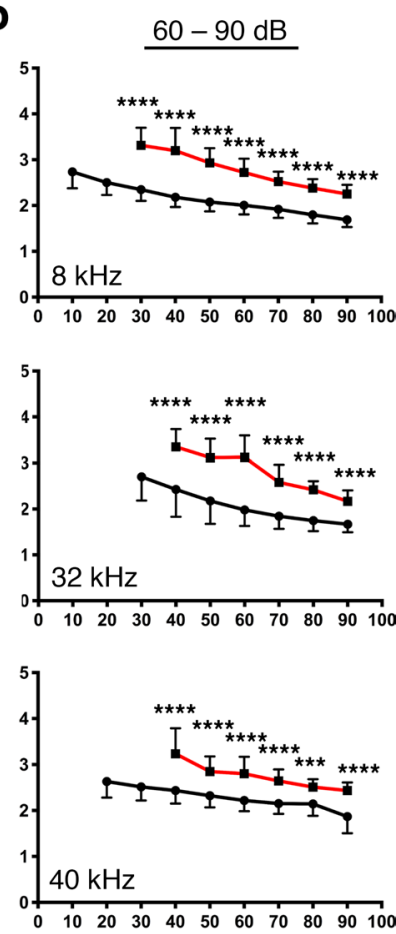

Stimulus (dB SPL)

Figure 3. Impaired wave I growth and latency in MCMV-infected mice. Wave I amplitude and latency of noninfected, control mice (black lines) compared with MCMV-infected mice (red lines) grouped according to ABR broadband click thresholds; 2 groups of animals that measured ABR thresholds between ( $A$ and C) 30-40 dB and (B and D) 60-90 dB are shown. Wave I amplitudes and latencies were measured from ABR tracings at indicated frequencies (8, 32, $40 \mathrm{kHz}$ ) and plotted against sound levels (dB SPL). $P$ values calculated using standard 2-tailed $t$ test. All error bars represent \pm SD from $n=15$ noninfected ears and $n=23$ MCMV-infected ears. ${ }^{* *} P<0.01 ;{ }^{* *} P<0.001 ;{ }^{* * *} P<0.0001$.

P32 mice was dissected and stained for Carboxy-terminal Binding Protein 2 (CtBP2), which labeled presynaptic ribbons found in IHC, and for GluR2, an AMPA receptor subunit that labeled postsynaptic glutamate receptors on afferent nerve terminals $(21,22)$. Low-magnification images were taken to measure cochlear length to convert cochlear regions to relevant cochlear frequencies. Subsequently, higher-magnification images were collected using confocal z-stacks to quantify the number of synapses per IHC (23). Regions of the cochlea corresponding to frequencies of $16 \mathrm{kHz}$ (apex) and $32 \mathrm{kHz}$ (mid) are shown, and synapses from additional images of regions corresponding to $40 \mathrm{kHz}$ (base) were quantified in cochlea from MCMV-infected mice with elevated ABR thresholds and noninfected control mice (Figure 5A). The number of CtBP2 puncta, GluR2-labeled terminals, and paired CtBP2/GluR2 ribbons and terminals were used to define the number of intact synapses (Table 1). The number of synapses was reduced in IHCs located in the apical and mid regions, but not in the basal region, in the cochlea from MCMV-infected mice with increased ABR thresholds as compared with cochleae from control animals with normal ABR thresholds (Figure 5A and Table 1).

To determine the impact of virus infection and inflammation on the integrity of innervation of $\mathrm{HC}$ in the cochlea, we imaged nerve fibers in the sensory epithelium of MCMV-infected and noninfected mice by immunostaining P32 cochlear wholemounts for neurofilament heavy chain (NF-H). As the density of innervation is different from the apex to basal regions of the cochlea, confocal z-stack images were taken from frequency-matched regions along the organ of Corti (21). In cochleae from MCMV-infected mice with elevated ABR thresholds, nerve fibers (Type II SGN) that directly innervate the OHCs were disorganized, with abnormal positioning of the nerve fibers when compared with those from noninfected, control mice (Figure 5B and Supplemental Figure 4, A and B; supplemental material available online with 
A
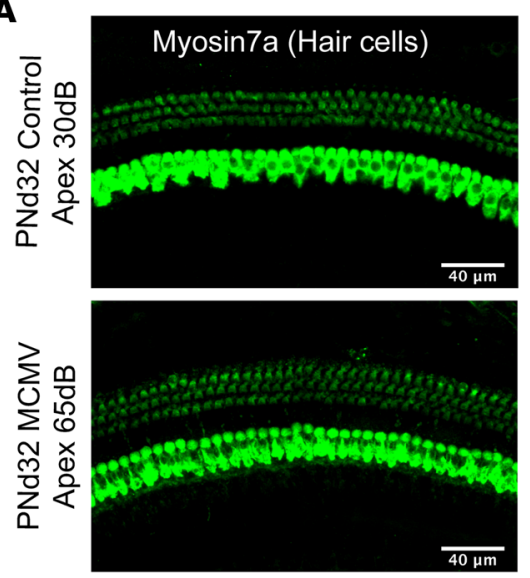
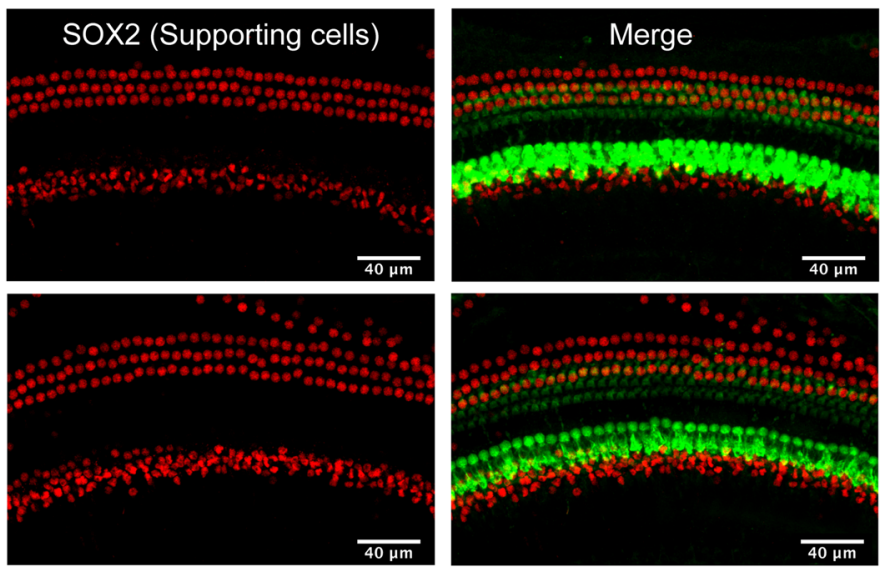

B

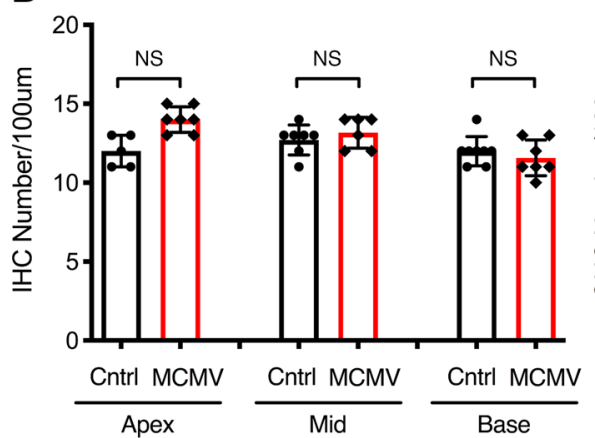

C

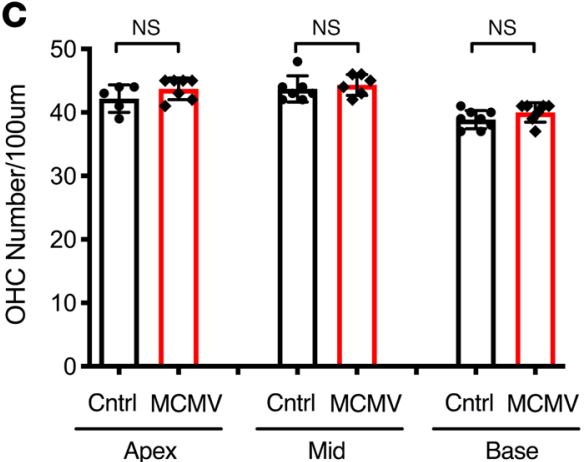

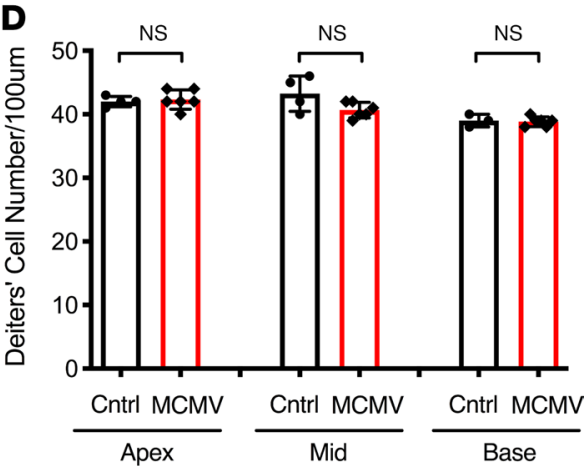

Figure 4. The number of sensory HCs and SCs in the organ of Corti are not altered by MCMV infection. (A) Cochleae were microdissected into 3 regions (apex, mid, and base) and immunostained for MyosinVIla, a marker for inner and outer hair cells, and Sox2, a marker for supporting cells. Z-stack images were acquired at 40x magnification and representative images from the apical region are shown. Scale bars: $40 \mu \mathrm{m}$. (B-D) Quantification of the total number of IHC (B), OHC (C), and SCs (Deiters' cells) (D) in the span of $100 \mu \mathrm{m}$ of the 3 regions displayed no significant differences in the noninfected and MCMV-infected cochlea. Data are shown as mean \pm SD, $n=4$ mice $/ n=8$ cochlea per experimental group. $P$ values calculated using 2 -tailed $t$ test. These results were derived from 2 independent experiments.

this article; https://doi.org/10.1172/jci.insight.128878DS1). In addition, the comparison of fluorescence intensities of NF-H under identical image acquisition settings suggested reduced density of Type I neurites around the basolateral region of the IHCs in cochleae of infected mice. The reduced density was more evident in Type II neurites innervating the OHCs in infected mice with elevated ABR thresholds compared with the noninfected control mice (Figure 5B and Supplemental Figure 4). When the total number of neurites innervating the $\mathrm{OHCs}$ in each region was quantified per length of epithelium, we noted a decrease in the overall number of neurite projections in infected mice with elevated $A B R$ thresholds as compared with control mice with normal ABR thresholds (Figure 5C).

Loss of SGNs in the cochlea of mice infected with MCMV. To further define the association between the loss of SGN and HL in MCMV-infected mice that we previously described, we quantified the density of SGNs in P32 mice infected as newborns but with different ABR thresholds (19). SGN soma quantification from all 3 regions of the cochlea (apex, mid, and base) revealed that the SGN density was decreased with increasing ABR thresholds (Figure 6). Although a loss of SGNs in MCMV-infected mice was present at all frequency regions of the cochlea in mice with ABR thresholds $>60 \mathrm{~dB}$, the loss of SGNs was more striking in the apex and mid-portions of the cochlea than in the basal region in mice with moderate increases in ABR thresholds, a distribution that was similar to the loss of IHC synapses as described above (Figure 5A, Figure 6C, and Table 1). Furthermore, there was a gradient of SGN loss from the apex to the base in infected mice with ABR click thresholds between $45-55 \mathrm{~dB}$ (Figure 6C). These results suggested that increasing impairment of auditory function in MCMV-infected mice, as measured by ABR, was also associated with increased loss of SGNs, raising the possibility of a common mechanism of damage following MCMV infection (19). 
A

Control (40 dB)

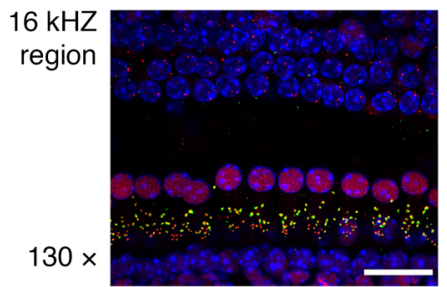

$00 \times$
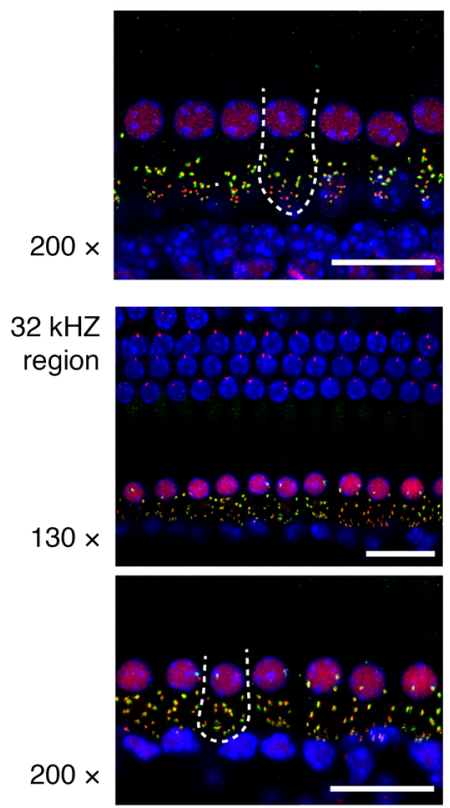

Infected (80 dB)
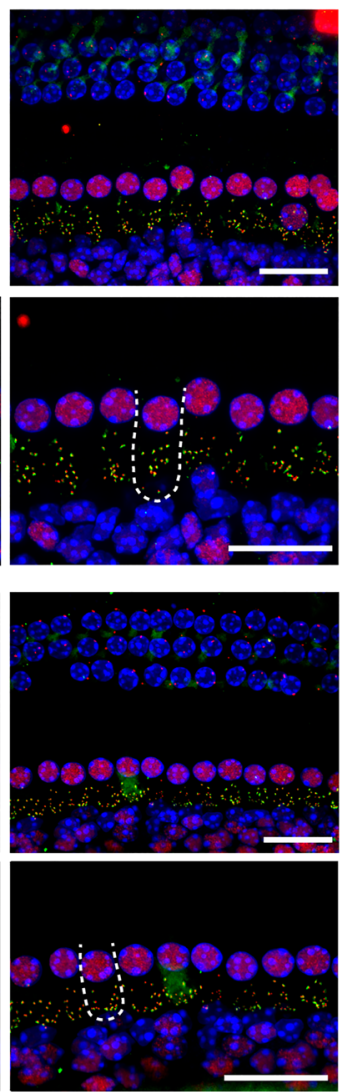

B

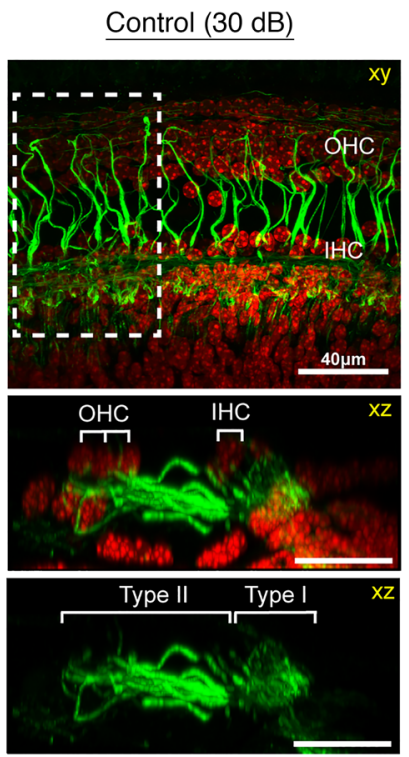

C
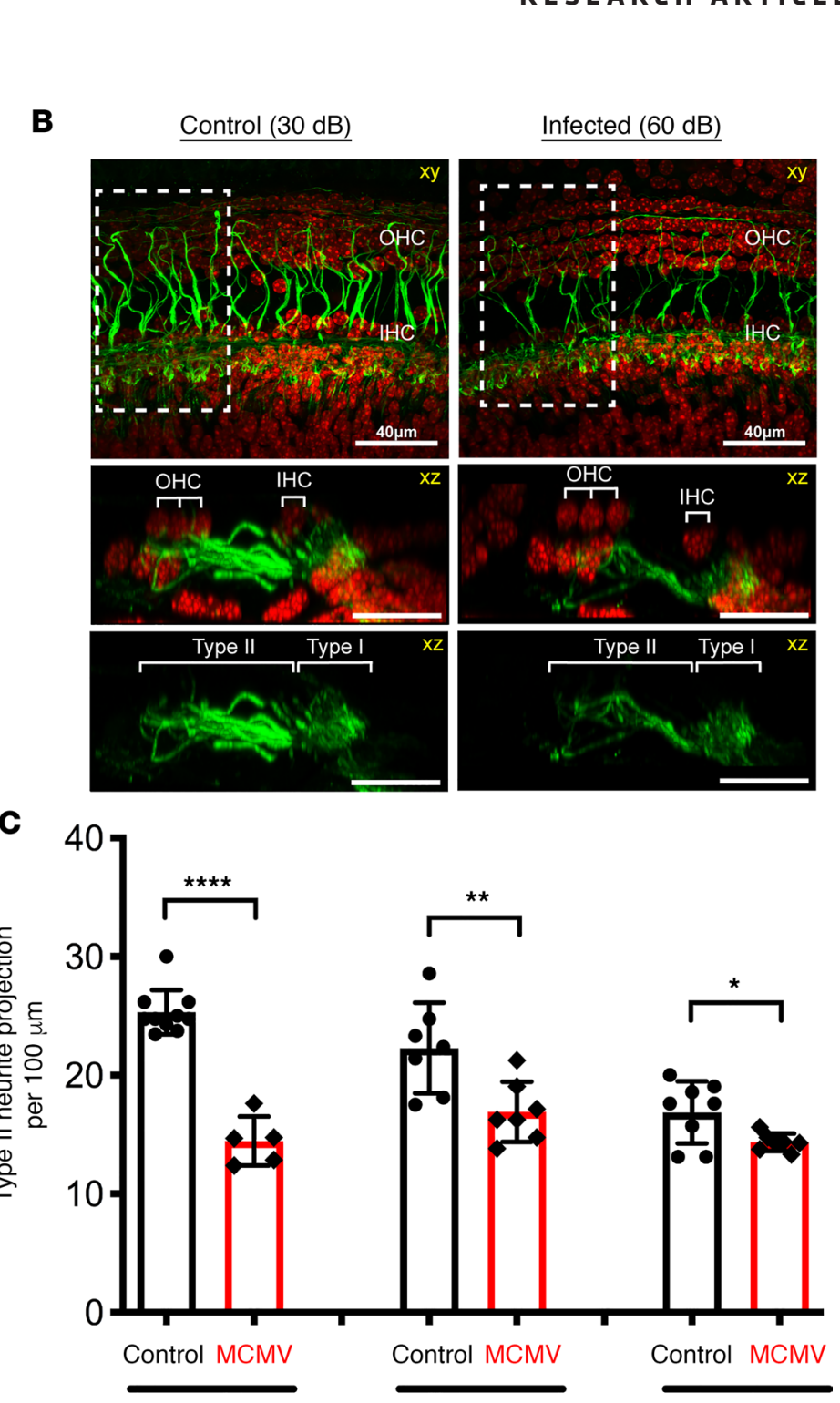

RESEARCH ARTIO

Infected (60 dB)
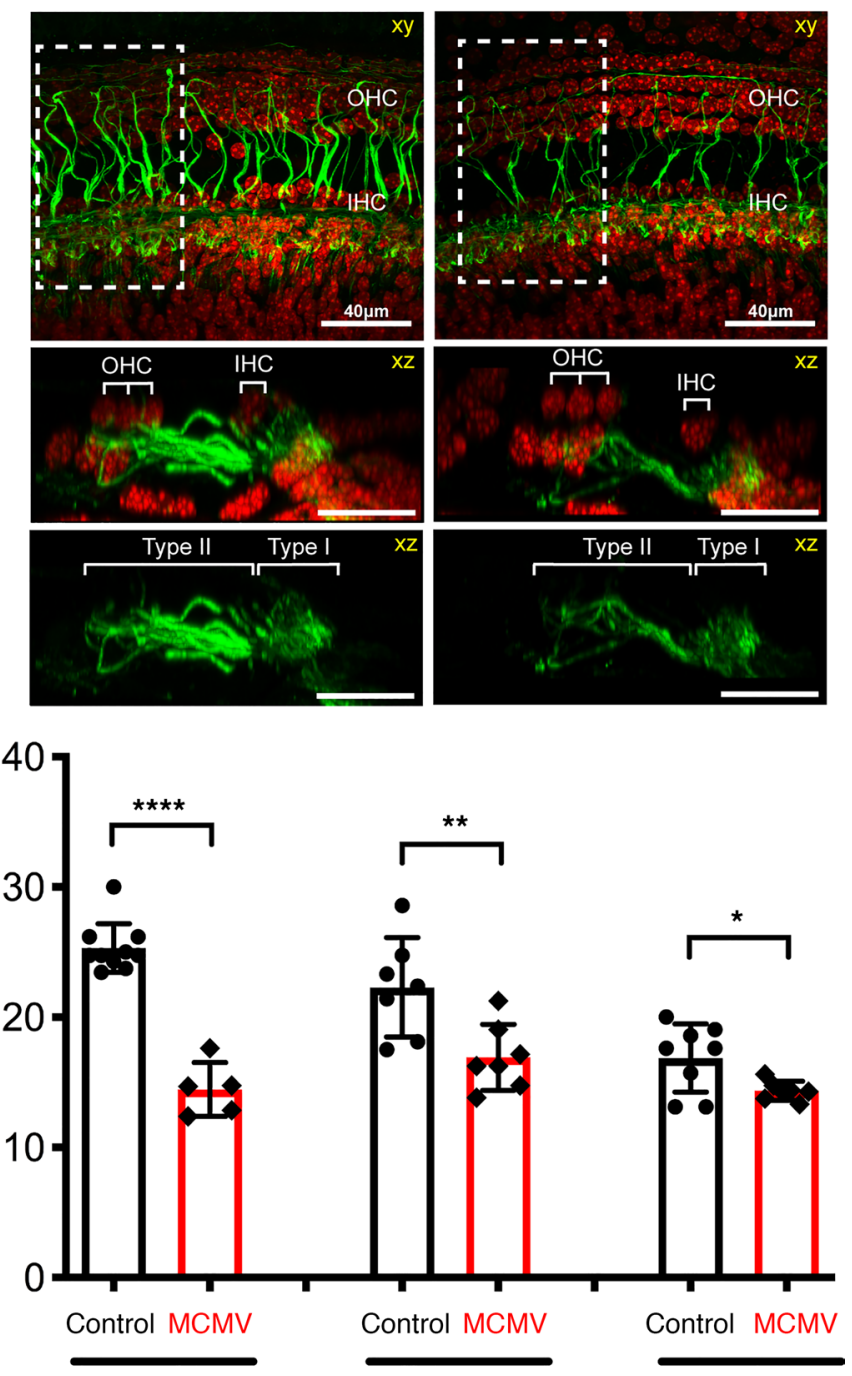

Mid

Base

Figure 5. MCMV infection results in reduced HC-SCN synapse density in the apex-middle region of the cochlea and disrupts morphology of SGN nerve fibers innervating sensory HCs. (A) Magnifications of 130x and 200x high-resolution confocal images were acquired by a z-stack series spanning the synaptic regions of the IHCs, and a series of z-stack images were merged, showing juxtaposed presynaptic ribbons (CtBP2; red) and postsynaptic receptors (GluR2; green) for P32 mice cochlear wholemounts. Hoechst (blue) was used to stain the nucleus. Representative images of apex (16 kHz) and mid ( $32 \mathrm{kHz}$ ) are shown in cochlea from a noninfected mouse and MCMV-infected (500 PFU) mouse with elevated ABR thresholds. White U-shaped outlines indicate a selected IHC and associated synapses. Note that anti-CtBP2 antibody also stains IHC nuclei. Scale bar: $20 \mu \mathrm{m}$ (B) Representative images of P32 mice cochlea immunostained for NF-H (green; neurofilament heavy chain) and Hoechst (red; nuclear stain) in the mid-apical (16 kHz) region of the cochlea. Top 2 panels are $x y$ projections, and bottom 4 panels are $x z$ projections displaying the cross-section of the field of white dashed line. Scale bar: $40 \mu \mathrm{m}$ (C) The number of Type II neurite projections, which innervate the OHCs, in the span of $100 \mu \mathrm{m}$ of the 3 regions displayed reduced numbers in MCMV-infected cochlea compared with the noninfected control. Data are shown as mean $\pm \mathrm{SD}, n=4-5$ mice $/ n=6-10$ cochlea per experimental group. $P$ values were calculated using a standard 2 -tailed $t$ test. These results were derived from 2 independent experiments. ${ }^{*} P<0.05 ;{ }^{* *} P<0.01 ;{ }^{* * * *} P<0.0001$.

Decreasing cochlear inflammation in MCMV-infected mice can limit early damage to the developing auditory system and impairment of auditory function. Previously, we have argued that MCMV-induced inflammation in the developing CNS and cochlea contributed significantly to altered neurodevelopment and HL in mice infected as newborns $(19,20,24)$. The levels of virus replication in P32 mice infected as newborns compared with their respective $A B R$ thresholds failed to reveal a correlation between viral load and the degree of auditory dysfunction, as measured by ABR (Figure 7, A and C). In contrast, when ABR thresholds were compared with the level of IFIT1 expression as a marker of cochlear inflammation, there was a significant correlation between the level of inflammation and ABR thresholds (Figure 7, B and D). Further evidence of the nonlinear relationship between cochlear inflammation and elevation of ABR thresholds was provided by 
Table 1. Quantification of CtBP2, GluR2, and paired CtBP2/GluR2 puncta associated with IHCs in the apex, middle, and base regions in the cochleae of noninfected, control, and MCMV-infected mice

\begin{tabular}{|c|c|c|c|c|c|c|c|c|c|}
\hline & \multicolumn{3}{|c|}{ CtBP2 } & \multicolumn{3}{|c|}{ GluR2 } & \multicolumn{3}{|c|}{ CtBP2/GluR2 } \\
\hline & Control & MCMV & $P$ value & Control & MCMV & $P$ value & Control & MCMV & $P$ value \\
\hline Apex & $17.236 \pm 0.6$ & $15.428 \pm 0.99$ & 0.0026 & $17.035 \pm 0.86$ & $15.821 \pm 0.79$ & 0.0013 & $16.507 \pm 0.97$ & $14.304 \pm 0.94$ & 0.0016 \\
\hline Base & $14.208 \pm 2.35$ & $13.500 \pm 1.14$ & 0.1206 & $14.067 \pm 2.29$ & $13.146 \pm 1.02$ & 0.1089 & $13.658 \pm 2.31$ & $12.917 \pm 1.01$ & 0.495 \\
\hline
\end{tabular}

the analysis of both ears from an individual MCMV-infected mouse that exhibited $>10 \mathrm{~dB}$ differences in $\mathrm{ABR}$ threshold between ears. In 5 mice meeting this criterion, we noted that, in 4 animals, the ear with the higher ABR threshold also had a higher expression of IFIT1 without a significant difference in the viral load in each ear (Figure 7E). Thus, when ABR thresholds from the right and left ears were used as a measure of damage to auditory pathways in individual animals, inflammation but not viral load remained associated with increased ABR threshold. Lastly, there was no correlation between cochlear virus load and the level of IFIT1 expression, suggesting that, although virus must be present in the cochlea to induce inflammation, parameters that govern the magnitude and perhaps the distribution of the host inflammatory responses in the virus-infected cochlea are not linearly related to total cochlear viral load (Supplemental Figure 1).

To determine if SGN loss occurred early after infection and during development of the auditory system, we measured the density of SGN soma from P8 noninfected, control and MCMV-infected mice $(25,26)$. Notably, SGN density in P8 cochleae was lower in all frequency regions in MCMV-infected mice compared with the noninfected, control mice (Supplemental Figure 2, A and B). In addition, there was no difference in the number of SGNs expressing cleaved Caspase 3 (Casp3a) in noninfected, control, and infected mice, suggesting that apoptosis was not a mechanism for loss of SGNs following MCMV infection (Supplemental Figure 2C).

To define the contribution of virus-induced inflammation to cochlear histopathology that was observed early after infection, we treated mice infected as newborns with methylprednisolone sodium succinate (pred), a corticosteroid with potent antiinflammatory activity, or vehicle alone from P3-P7 and harvested cochleae on P8 (Supplemental Figure 3A). Treatment with pred did not alter the quantity of virus in the cochlea but decreased the expression of the ISG, IFIT1, in the cochlea (Figure 8, A and B). We next analyzed the effect of pred treatment during early postnatal development on the MCMV-induced SGN loss, as this histopathologic measure of virus-induced damage reflected the impact of MCMV infection on the cochlea both early in development and at later time points when auditory pathways are mature (19). Treatment with pred significantly reduced SGN loss in P8 MCMV-infected animals, as compared with infected animals treated with vehicle alone (Figure 8C). Notably, the SGN densities in the apex and mid-portion of the cochlea in pred-treated animals were not significantly different than the SGN density in noninfected, control animals treated with vehicle alone (Figure 8C). These results showed that virus-induced inflammation contributed to histopathological findings in the developing cochleae that were observed shortly after MCMV infection in newborn mice.

The results described above raise the possibility that dampening virus-induced inflammatory responses during early cochlear development could improve auditory function in infected mice. To explore these possibilities, noninfected, control mice and MCMV-infected mice were treated with vehicle or pred utilizing a similar protocol as described above, but treatment was extended through P20 with a short taper of dosage beginning on P17 (Supplemental Figure 3B), thus providing an antiinflammatory effect of pred during resolution of cochlear inflammation (Figure 1C). Importantly, the ABR thresholds of noninfected, control mice following prolonged treatment with pred did not differ from the noninfected, control group treated with vehicle only, indicating that pred treatment did not alter auditory function in noninfected, control mice (Figure 9A). However, prolonged treatment of infected mice with pred resulted in a significantly increased mortality rate, as a group of pred-treated MCMV-infected mice had a small but significant improvement in the number of ears with normal ABR thresholds $(<40 \mathrm{~dB})$ compared with infected mice treated only with vehicle (Figure 9A). 
A

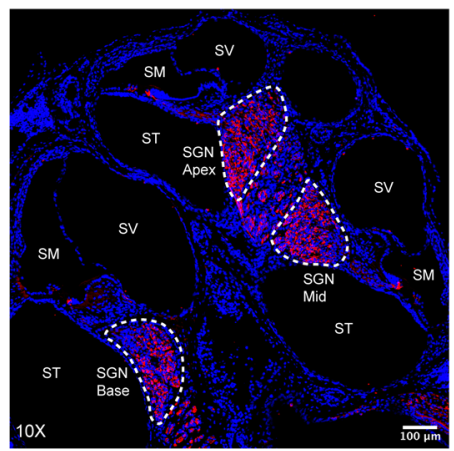

B
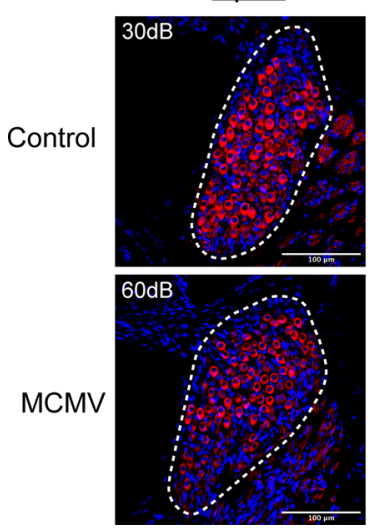

Mid
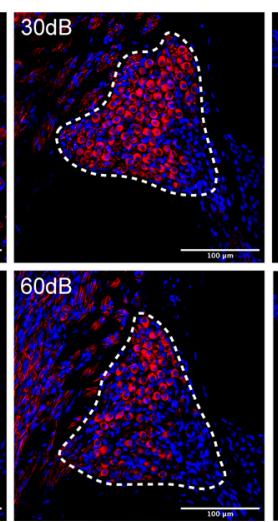

C
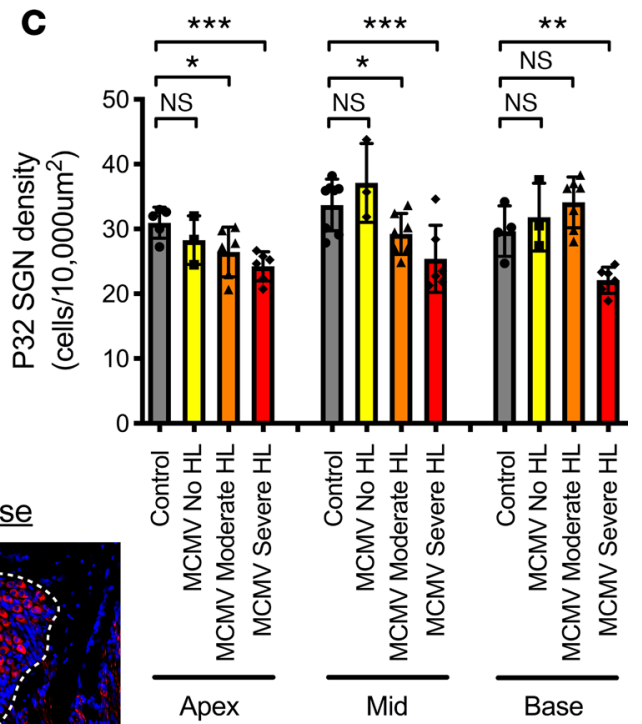

Figure 6. ABR elevation in MCMV-infected mice is associated with the level of SGN loss. P32 sections from noninfected, control and MCMVinfected (500 PFU) mice were stained for Tuj-1 to detect SGNs. (A) A 10x image of cochlea stained for Tuj-1 displaying the 3 regions (apex, mid, and base) of the Rosenthal's canal. SV, Scala Vestibuli; SM, Scala Media; ST, Scala Tympani; SCN, Spiral Ganglion Neuron. (B) Representative images (40x) of SCNs of P32 cochlea from infected mice with elevated ABR threshold (60-90 dB) demonstrate reduced number of SGN soma compared with the noninfected control cochlea with normal ABR thresholds ( $30-40 \mathrm{~dB})$. (C) Cochleae from P32 mice were grouped according to their ABR click thresholds. SGNs were quantified and normalized to the areas of Rosenthal's canal to provide SGN density (cells $\left./ 10,000 \mu \mathrm{m}^{2}\right)$ in the 3 regions of the cochlea. SGN quantification data of cochlear sections are shown as mean \pm SD, $n=3$ mice/ 6 cochlea per experimental group. $P$ values were calculated using 1-way ANOVA with Tukey's multiple comparison's test. Data are representative of 2 independent experiments. Scale bars: $100 \mu \mathrm{m}$. ${ }^{*} P<0.05 ;{ }^{* *} P<0.01 ;{ }^{* *} P<0.001$.

To further define the impact of pred treatment on observed abnormal auditory functions in MCMV-infected mice, we determined the growth of the ABR wave I amplitude and latency at 8 and $32 \mathrm{kHz}$ in control mice and MCMV-infected mice treated with either vehicle or pred. Treatment of infected mice with pred normalized wave I amplitude growth and latency when compared with infected mice treated with vehicle alone (Figure 9, B-E). Moreover, there was no difference in wave I amplitude growth and latency between pred-treated infected mice with elevated ABR thresholds and vehicle-treated but noninfected, control mice (Figure 9, B-E). Together, these data show that a reduction in virus-induced inflammation during cochlear development led to measurable improvements in auditory function in young adult mice and provide support for the hypothesis that inflammation and not lytic virus infection contributed to the altered function of auditory pathways in newborn mice infected with MCMV.

To determine if the impact of pred treatment on auditory function was also reflected in the preservation of SGN, thus providing a histopathological correlate of the impact of pred treatment on these mice, SGN soma density was quantified in MCMV-infected mice or control mice treated with pred or vehicle on P34 following ABR testing. Similar to our previous findings, the quantification of SGNs demonstrated a decreased density of SGNs in vehicle-treated MCMV-infected mice as compared with vehicle-treated, noninfected mice in all 3 regions of the cochlea (Figure 10). Importantly, pred treatment of infected mice prevented SGN loss when compared with infected mice treated with vehicle only, thus providing further evidence that MCMV-induced inflammation was at least one mechanism of damage of the cochlea in MCMV-infected mice (Figure 10). 
A

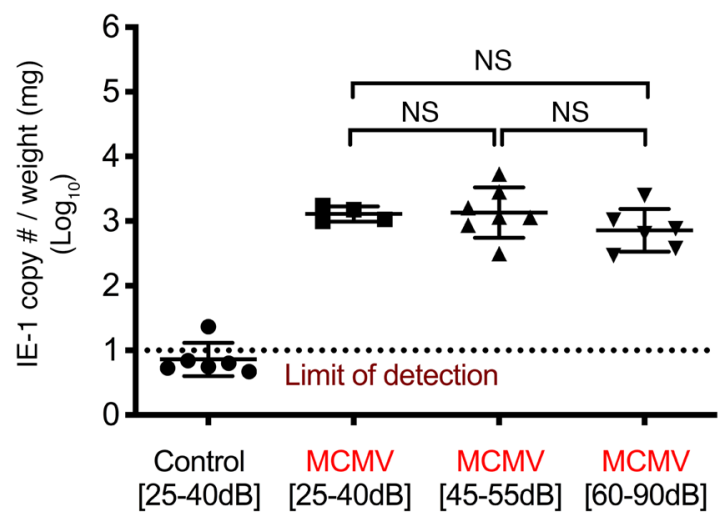

B

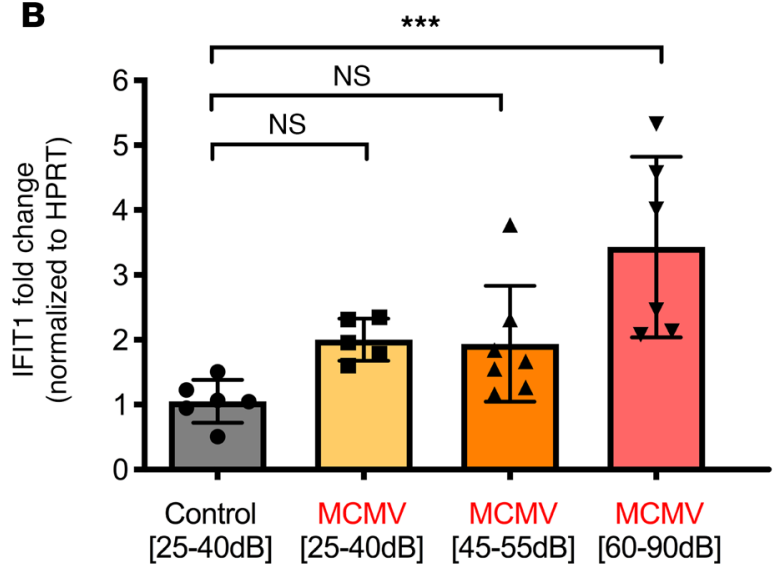

C

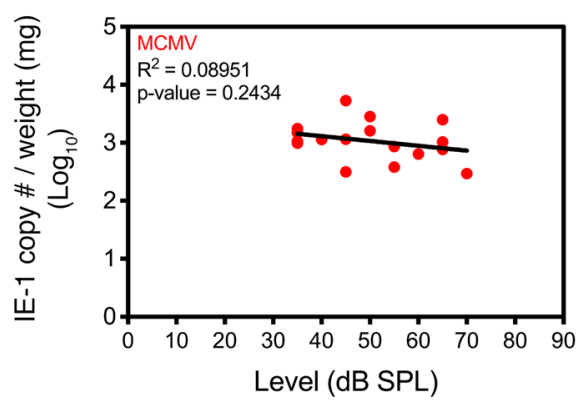

D

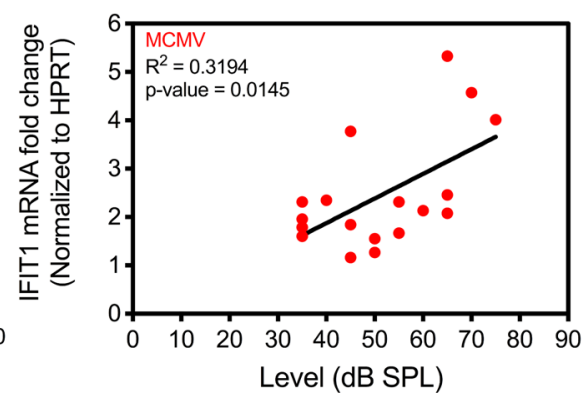

E

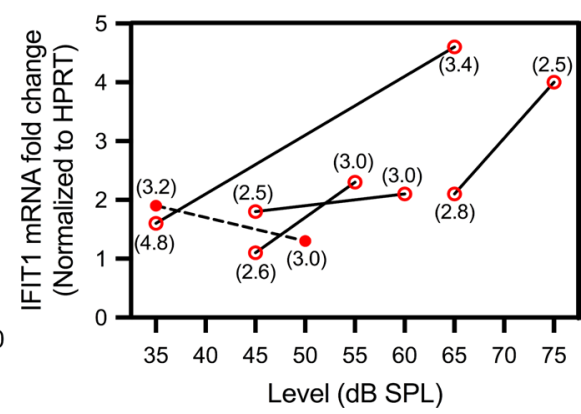

Figure 7. Cochlear inflammation, and not viral load, in MCMV-infected mice correlates with ABR threshold. (A) MCMV genome copy number/mg cochlear tissue is plotted vs. ABR threshold of individual mice. (B) IFIT1 expression plotted against the ABR threshold in individual mice. The differences between groups are determined with 1-way ANOVA with Tukey's multiple comparison's test. (C and $\mathbf{D})$ The correlation between the cochlear viral load (C) or IFIT1 expression (D) and ABR thresholds are shown. Linear regression analysis was performed, and $P$ value, as a test for linear trend, is shown. Note that only the correlation between IFIT1 expression and ABR thresholds $(\mathbf{D})$ reached statistical significance $(P=0.0145)$. $(\mathbf{E})$ Expression of IFIT1 and viral load from individual infected mice that displayed more than $10 \mathrm{~dB}$ difference in ABR thresholds between right and left ear were determined. ABR thresholds from individual mice were plotted against IFIT1 fold change, and viral load (genome copy) is shown in parentheses adjacent to each data point. Data points (red open circles) representing cochleae from individual mice are connected by a solid line indicating positive correlation, while data points (red close circles) connected by dashed line indicate negative correlation between ABR threshold and IFIT1 fold change. Five mice with unilateral HL were analyzed from total experimental group of $9 \mathrm{MCMV}$-infected mice with elevated $A B R$ thresholds. ${ }^{* * *} P<0.001$.

\section{Discussion}

SNHL remains the most common long-term sequelae of cCMV infection, yet mechanisms leading to SNHL in infected infants are undefined $(7,27)$. Much of the histopathology described in studies of temporal bones from fetuses infected in utero with HCMV has been recapitulated in this murine model of cCMV-associated HL, and infected mice exhibit a clinical spectrum of SNHL similar to that described in cCMV-infected infants $(1,7,19)$. Furthermore, in this model, significant variability is observed in the level of ABR threshold elevation in individual animals, a well-described observation in clinical studies of SNHL in infants with cCMV. Notably, the variation in the severity of HL in this model can be observed when littermate mice are inoculated with equivalent amounts of a molecularly cloned virus, suggesting that multiple mechanisms could contribute to CMV-induced SNHL. Although this mouse model does not recapitulate intrauterine transmission of virus, when infected, newborn mice (P0) are neurodevelopmentally and immunologically similar to early second trimester human fetus, and auditory maturation in mice is not complete until about the third postnatal week, with the onset of hearing between P12-P14. Thus, in this model system, virus infection in the cochlea takes place during early cochlear development, including a critical period for connectivity of neural pathways.

We cataloged inner ear histopathology in young adult mice (P32-P34) infected in the newborn period with evidence of moderate to severe elevations in ABR thresholds. Although we could readily demonstrate elevated ABR and DPOAE thresholds in a subset of the infected mice, we found no evidence of viral infection 
A

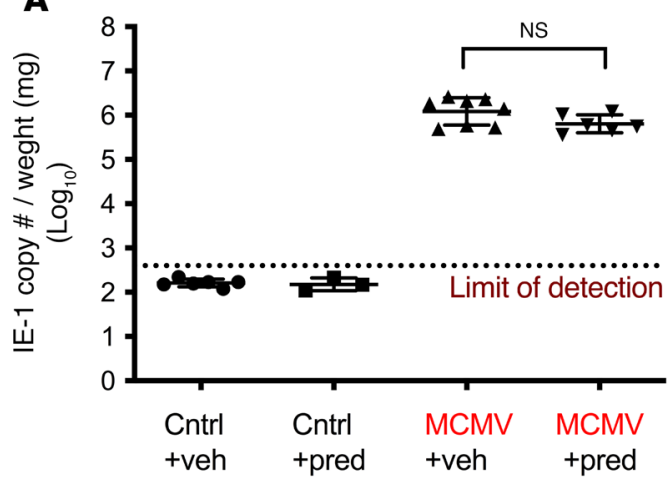

B

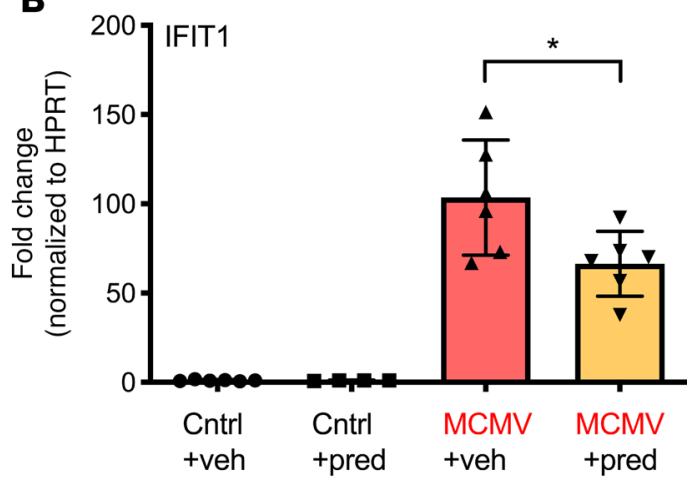

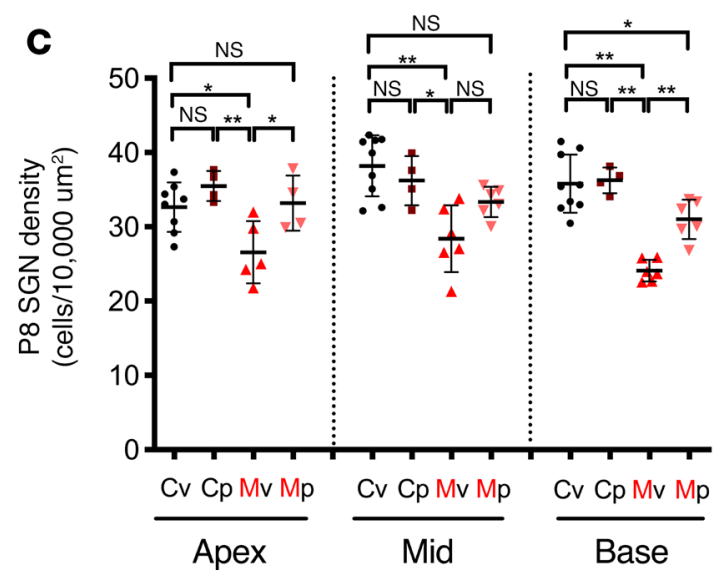

Figure 8. Prednisolone treatment decreases cochlear inflammation and loss of SGNs in P8 mice infected with MCMV. (A) MCMV DNA is quantified and represented as log genome copy/mg cochlear tissue. Note that pred treatment did not affect MCMV replication in the cochlea. (B) The impact on the expression of IFIT1 in cochlea of pred-treated, infected animals is shown. Note significant differences between the means of the cochlea from MCMV-infected mice vs. those from pred-treated MCMV-infected mice. (C) SGNs were quantified in 3 regions of cochlea. SGN density were plotted for noninfected, control mice given only vehicle (Cv), noninfected, control mice given pred (Cp), MCMV-infected mice treated with only vehicle (Mv), and MCMV-infected mice treated with pred (Mp). Medians were analyzed by 1-way ANOVA (Kruskal-Wallis test) with Dunn's post test comparisons test. ${ }^{*} P<0.05 ;{ }^{* *} P<0.01$.

in the sensory epithelium, nor evidence of significant damage of the sensory epithelium, including a loss of HCs in mice with elevated thresholds. These findings were in striking contrast to results from murine models utilizing intracerebral (i.c.) inoculation of MCMV in which i.c. infection of newborn mice with MCMV consistently produces a significant loss of the sensory epithelium and corresponding elevations of ABR and DPOAE thresholds in almost all infected animals (28-31). The route of viral spread to the inner ear following i.c. injection vs. that following hematogenous spread is distinctly different and could account for differences in experimental findings in these different models (28-31). It was of interest that we observed a gradient of SGN loss from apical to basal turn with more neuronal loss in the apical region compared with the basal region. In contrast, young rodents inoculated with bacteria into the cisterna magna to produce meningitis exhibited the most significant loss of SGN in the basal turn of the cochlea, with loss of SGN in the middle and apical turns being observed only in animals with significant $\mathrm{HC}$ loss and ABR thresholds $>90 \mathrm{~dB}$ (32). Similarly, we observed a gradient of loss of IHC synapses in mice with elevated ABR thresholds, with more loss of synapses in the apical region of the cochlea, whereas the basal region was more affected in infant rats with bacterial meningitis (32). These different animal models of inner ear infection leading to HL suggest different routes of microbial spread, resulting in differential susceptibilities of the apical and basal regions of the cochlear duct. In these models of $\mathrm{HL}$ induced by intracisternal inoculation of bacteria, it has been argued that bacteria as well as the resultant inflammatory response spread from the cerebrospinal fluid (CSF) to the cochlea via the cochlear aqueduct (32). Our findings suggest that hematogenous spread of virus in mice infected peripherally with MCMV results in a different route of virus entry into the cochlea. 

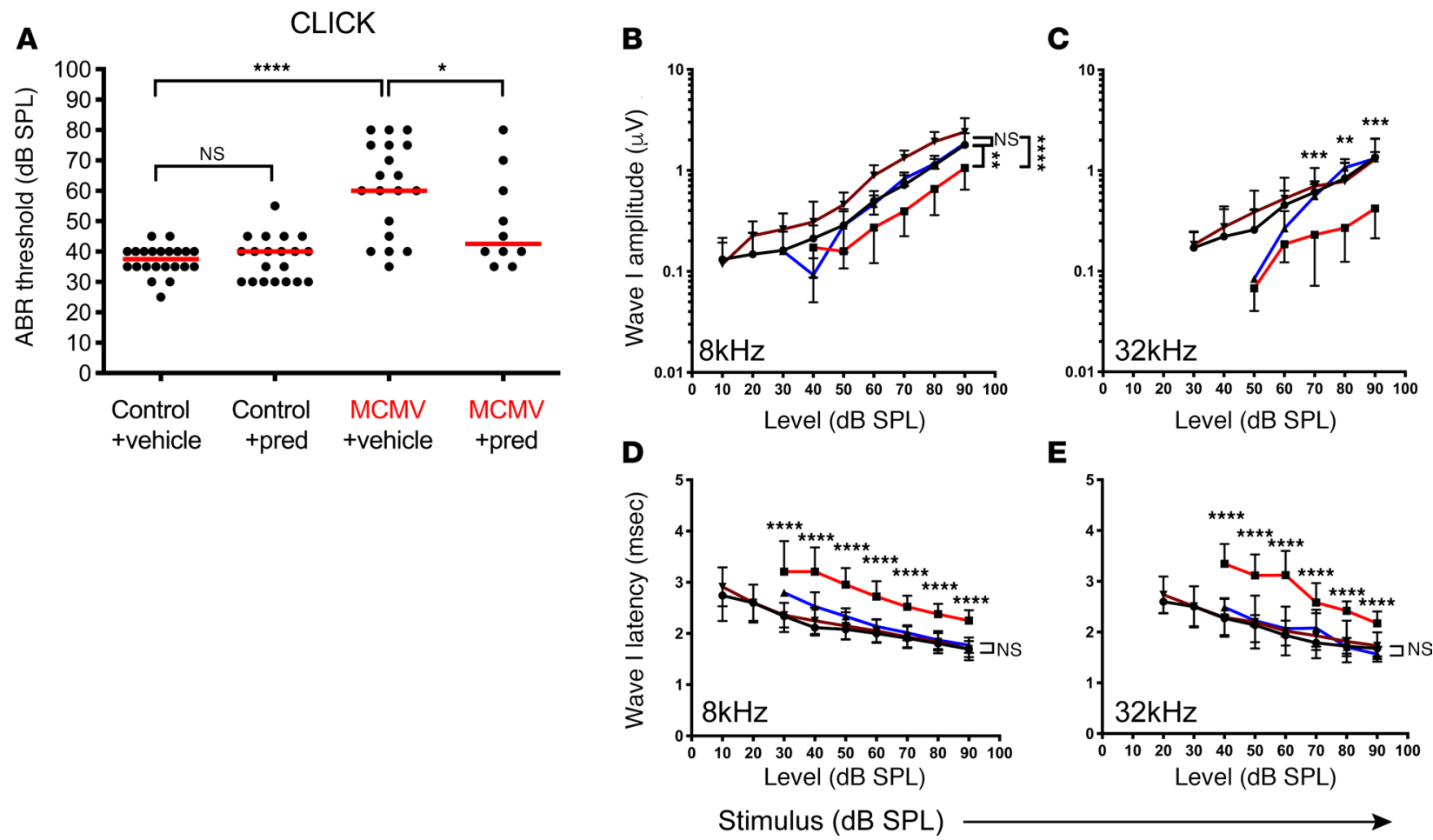

Figure 9. Treatment of MCMV-infected mice with methylprednisolone improves auditory function. (A) Noninfected, control mice and mice infected with MCMV (500 PFU) as newborns were treated with vehicle (saline) alone or methylprednisolne (pred) between P3-P20, and ABR measurements were performed on P32 mice. For each experimental group, the number of ears was used as shown: Control+vehicle ( $n=22$ ears), Control+pred ( $n=20$ ears), MCMV+vehicle ( $n=19$ ears), and MCMV+pred ( $n=10$ ears). Results are expressed as medians (red bar), and statistical comparisons are made by 1-way ANOVA with Tukey's multiple comparisons test. (B-E) Wave I amplitudes (B and $\mathbf{C}$ ) and latencies ( $\mathbf{D}$ and $\mathbf{E})$ from noninfected, control mice given vehicle (black tracing); noninfected, control mice given pred (brown tracing); MCMV-infected mice given vehicle (red tracing); and MCMV-infected mice pred (blue tracing) were determined. Results are expressed as means \pm SD, and statistical comparisons were made by 1-way ANOVA with Tukey's multiple comparisons test. ${ }^{*} P<0.05 ;{ }^{* *} P<0.01 ;{ }^{* *} P<0.001 ;{ }^{* * *} P<0.0001$.

We detected only widely scattered small foci of MCMV-infected cells in the modiolus and the stria vascularis in P8 mice infected as newborns - findings inconsistent with a mechanism of cochlear damage secondary to direct virus-mediated cytopathology (Figure 1B) (19). Furthermore, we documented the expression of the ISGs and proinflammatory cytokines in the cochlea early after infection, as well as dense but evenly distributed Iba- $1^{+}$myeloid cells throughout the cochlea (Figure 1B). Thus, it is possible that unregulated host inflammatory responses to viral infection in the cochlea leads to pathology and HL, as has been suggested in other studies (28-32). Thus, in models using i.c. MCMV inoculations, it could be argued that direct damage to the inner ear sensory epithelium could mask important effects of cochlear inflammation that are in addition to the direct cytolytic effects of viral infection in these models. This possibility was again illustrated by results from a model of $\mathrm{HL}$ associated with bacterial meningitis in young rats in which elevation of ABR thresholds and damage to the sensory epithelium could be directly related to both the size of the bacterial inoculum and intensity of inflammatory response in the cochlea, as measured by the expression of TNF- $\alpha$ (32). Although these data argue for a direct role of cochlear inflammation in HL, this interpretation was also confounded by significant HC loss associated with higher bacterial inoculums, raising the possibility that direct effects of the bacterial infection was the primary cause of $\mathrm{HL}$ in these animals, and not inflammation (32). In our experiments, we did not detect damage or HC loss in the sensory epithelium, but there was a significant inflammatory response induced by CMV. When viewed together, these findings suggested that expression of proinflammatory cytokines such as TNF- $\alpha$ could be an important contributor to HL, irrespective of the preservation of HCs. Finally, with the exception of damage to the sensory epithelium, there were similarities in the histologic findings following both routes of infection, in that both peripheral and intracranial routes appeared to result in the loss of synapses on IHCs and SGNs (32). Thus, the characteristics of microbe-induced inflammatory responses in the cochlea during hearing development could define the mechanisms of auditory dysfunction that develops in the absence of loss or destruction of the sensory epithelium. 


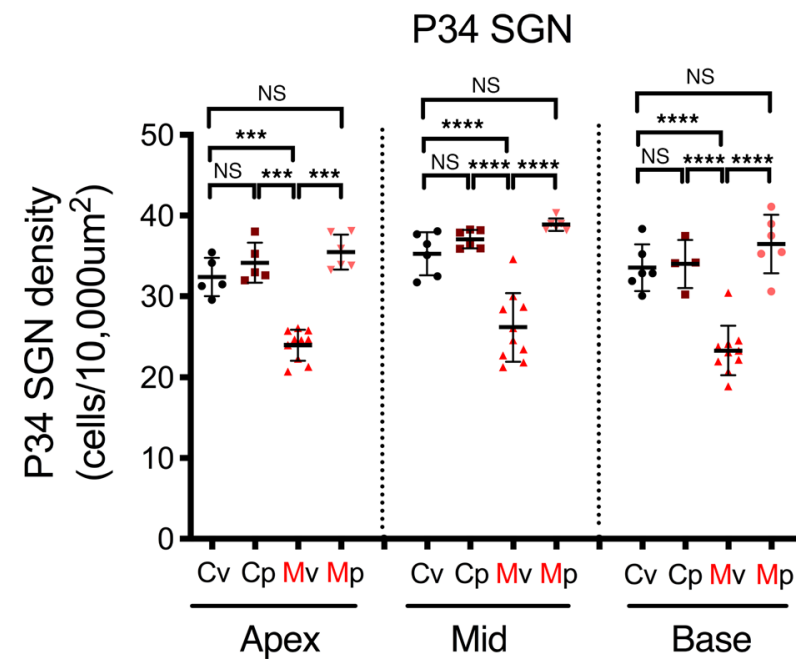

Figure 10. Treatment with methylprednisolone limits SGN loss in MCMV-infected mice. Noninfected, control or MCMV-infected (500 PFU) mice were treated with vehicle alone or methylprednisolne (pred) as described in Figure 9. Mice were harvested on P34, and the SGN density was determined. Quantification of SGN density shows the impact of pred treatment on preservation of SGNs in MCMV-infected mice. Medians were analyzed by 1-way ANOVA (Kruskal-Wallis test) with Dunn's comparisons test. For each experimental group, the number of ears was used as shown: Control+vehicle (Cv; $n=6$ ears), Control+pred (Cp; $n=6$ ears), MCMV+vehicle ( $n=10$ ears), MCMV+pred ( $n=6$ ears). ${ }^{* *} P<0.001$; ${ }^{* * *} P<0.0001$.

Although we have not defined a mechanism for elevated ABR thresholds in this model of HL following virus infection of the cochlea, defective sensory cell function has been shown to result in elevated ABR and DPOAE thresholds in spite of morphologically normal IHCs and OHCs. There are numerous examples of mouse mutants in which HC are present and appear morphologically normal by light microscopy, yet affected mice have elevated ABR thresholds, such as in the VGLUT3-null mouse (33). Another plausible mechanism that could explain the elevated ABR thresholds associated with MCMV infection is damage to the stria vascularis, resulting in alterations in the endocochlear potentials. Studies have suggested that viral- or bacterial-induced inflammation can disrupt vascular integrity in the stria vascularis, leading to leaky blood labyrinth barrier and imbalance of the ion composition in the endolymph (34). Potentially, proinflammatory cytokines in the MCMV-infected cochlea could alter permeability of the intrastrial blood labyrinth barrier (19). Thus, additional studies in this model could provide insight into mechanisms that contribute to inflammation-induced HC dysfunction and, ultimately, elevated ABR thresholds following viral infection of the cochlea.

Previously, we have demonstrated a significant contribution of host innate immune responses to altered brain development in this model of virus infection of the developing CNS $(20,24)$. Inhibition of host responses, by treatment with either pred or TNF- $\alpha$ neutralizing antibodies, normalized brain development in infected mice without significantly impacting virus replication $(20,24)$. A protocol similar to that used in these earlier studies was initially utilized to inhibit cochlear inflammation in infected mice, but results from several pilot experiments indicated that higher doses of steroids were required to decrease virus-induced inflammation in the cochlea. This finding suggested several possibilities, including (a) the disposition of pred in the developing cochlea was different than in the brains of infected mice, (b) virus-induced inflammation was more robust in the cochlea than the brain, or (c) areas of focal infection and inflammation in the cochlea were not readily accessible to corticosteroids delivered systemically. Consistent with these possibilities was the observation that, even though increased dosages of pred decreased the inflammatory responses in the cochleae of infected mice, the reduction in the expression of IFIT1 in the cochlea was about 3-fold less than that achieved in the cerebella of P8 MCMV-infected mice treated with a significantly lower dosages of pred (20). Nonetheless, the decrease in cochlear inflammation that followed pred treatment was associated with improvement, albeit very modest, in ABR thresholds when measured with broadband clicks, a decrease in SGN loss, and functional improvement in the growth of the amplitude and latency of wave I in ABR testing. Future modifications of our treatment protocols will be aimed at more robust reductions in MCMV-induced cochlear inflammation, as we believe that HC dysfunction in this model system could be directly related to the level of inflammation. 
MCMV-infected mice with significant elevations of ABR thresholds demonstrated poor growth in wave I amplitude and delays in wave I latency when compared with noninfected, control mice. It is known that loss of IHC synapses can produce this effect $(21,35,36)$. We have shown that the IHC synapses were fewer in number and had more unpaired contacts in MCMV-infected mice than in age-matched control mice. Furthermore, MCMV-infected cochleae revealed an overall disorganization and decreased density of Type II SGN nerve fibers, and presumably Type I nerve fibers, as suggested by a decreased density at the basolateral orientation of the IHCs (Figure 5B). It is important to stress that, without the use of antibodies against specific markers such as peripherin, we could not quantify the relative loss of Type I and Type II nerve fibers in this study; however, we noted that, overall, there was a uniform decrease in neurites in the apex, mid, and base of cochleae from infected mice as compared with controls. Overall, our findings including decreased wave I amplitude, reduced synapses, and damaged SGN nerve fibers suggest auditory neuropathy as a potential component of MCMV-induced cochlear dysfunction. However, it is important to note that, in many model systems, SGN cell number can be significantly reduced with a notable loss of IHC synapses, yet normal ABR thresholds are maintained (21). Thus, it is unlikely that the loss of SGNs and IHC synapses alone is sufficient to explain the ABR threshold shifts that we observed in 50\%-60\% of MCMV-infected mice, indicating that the mechanism of ABR threshold shifts in these infected mice remain incompletely defined.

In summary, we have described cochlear histopathology that could contribute to the impaired auditory function in young adult mice infected with MCMV. The focal nature of the infection, coupled with a robust virus-induced cochlear inflammation early in development, showed that virus-induced immunopathology, and not direct virus cytopathic effects, was a proximal mechanism of disease in this model of SNHL associated with cCMV infection. Evidence for this mechanism of disease in this model was obtained by limiting cochlear inflammation in infected mice with corticosteroids. This treatment resulted in a decrease in the histopathological findings in the cochlea and minimal but demonstrable improvement in auditory function. Thus, microbe-induced immunopathology that follows cochlear infection appears to represent a common thread between findings from this model of cCMV-associated HL and those from models of HL following bacterial meningitis early in auditory development (32). Importantly, deficits in ABR thresholds were observed in the absence of overt loss and/or morphological damage of HC in our study, suggesting that defining the nature of inflammatory responses that lead to elevated ABR and DPOAE thresholds could provide critical insight into the mechanisms of HL associated with CCMV infection and potentially further define mechanisms of HL that are associated with other CNS infections.

\section{Methods}

Animals, MCMV infection, and treatment with methyl prednisolone. Pathogen-free BALB/c mice were purchased from Charles River Laboratories. Virus stocks were derived by reconstitution of a molecular clone of Smith MCMV (MCMV-repaired) that has been shown to be fully repaired by sequence analysis and virulence in vivo. Infectious virus was expanded in M2-10B4, a mouse BM stromal cell line (ATCC, CRL-1972), and passaged $<3$ times $(37,38)$. Infectious virus titers of viral stocks were determined by performing viral plaque assay using mouse embryonic fibroblast (MEF) and tittered in vivo to establish inoculum size for consistent induction of $\mathrm{HL}$ in mice. Aliquoted virus stocks were stored in $-80^{\circ} \mathrm{C}$ until used to infect animals. Newborn mouse pups, both male and female, were infected with 200 or 500 PFU of MCMV-repaired virus in sterile DPBS. Virus inoculums were given by i.p. injection into newborn BALB/c mice within 12 hours following birth, as described (39). Pregnancies were not timed but produced by cohousing of a single male with 2 female mice, and pregnant mice were observed daily for delivery.

Corticosteroid treatment. In developing animals, control and MCMV-infected pups were treated on P3-P7 by i.p. injection with pred at $18 \mathrm{mg} / \mathrm{kg}$ in saline $(0.9 \% \mathrm{NaCl})$. Treatments were administered once per day, and mice were sacrificed at P8 and within 12-16 hours after the last injection. As a control, noninfected and MCMV-infected animals were given $50 \mu 1$ of saline once per day on P3-P7. For long-term treatments, pred was injected at $18 \mathrm{mg} / \mathrm{kg}$ daily on P3-P16, $9 \mathrm{mg} / \mathrm{kg}$ on P17-P18, and $4 \mathrm{mg} / \mathrm{kg}$ on P19-P20 to taper steroid withdrawal in treated mice.

Virus genome copy number quantitation/titer analysis and gene expression. Mice were sacrificed at various time points, exhaustively perfused with PBS through the left ventricle, and following perfusion, organs including bony labyrinth were harvested. Following isolation of the bony labyrinth (cochlea and vestibular organ), any remaining bone or tissue was carefully and completely removed and DNA/RNA were isolated $(n=$ 8-10) using E.Z.N.A Total RNA Kit I (Omega Bio-tek) with modifications in the manufacturer's protocol to 
analyze viral genome copy number by quantitative PCR (qPCR). qPCR (TaqMan Gene Expression; Thermo Fisher Scientific) was based on amplification of exon 4 of the viral immediate early-1 (IE-1) gene using forward primer (5'-GGC TCC ATG ATC CAC CCT GTT A-3') and reverse primer (5'-GCC TTC ATC TGC TGC CAT ACT-3'), and the probe (5'-AGC CTT TCC TGG ATG CCA GGT CTC A-3') was labeled with reporter dye FAM and quencher dye TAMRA. Serial dilutions of plasmid with cloned MCMV IE-1 exon 4 PCR product were used to generate the standard curve for quantification of viral genome copy number. Samples were run in duplicate, and viral genome copy numbers are expressed as $\log _{10}$ genome copies per $\mathrm{mg}$ of tissue. In preliminary studies, the viral genome copy number per mg of tissue derived from cochlea and vestibular apparatus dissected from the bony labyrinth from P8-infected mice were comparable with the viral genome copy number per mg of tissue when the total bony labyrinth (vestibular apparatus plus cochlea) was extracted. In contrast, in these same mice, the expression of IFIT1 was approximately 2 -fold higher per mg of tissue from the cochlea when compared with the vestibular apparatus.

For quantification of RNA transcripts, total RNA was isolated from bony labyrinth using E.Z.N.A Total RNA kit (Omega Bio-tek) with modifications of the manufacturer's protocol. Invitrogen Superscript III First strand synthesis (Thermo Fisher Scientific) was used to synthesize cDNA from total RNA. TaqMan gene expression master mix was used to detect HPRT (Mm00446968_m1), IFIT1 (Mm00515153_ $\mathrm{m} 1$ ), and TNF (Mm00443258_m1) (Invitrogen). The FAM and MGB were used as the reporter dye and the quencher, respectively. HPRT was used as an internal control gene, and fold change was expressed as $2^{-\Delta \Delta C t}$. Values of experimental groups were normalized to values derived from the noninfected, control group.

ABR measurements. ABRs were measured in P32-P40 noninfected and MCMV-infected mice, as well as in mice treated with vehicle or pred. Mice were anesthetized i.p. with ketamine $(50 \mathrm{mg} / \mathrm{kg})$ and medetomidine $(0.5 \mathrm{mg} / \mathrm{kg})$. Anesthetized animals were maintained at $37^{\circ} \mathrm{C}$ during $\mathrm{ABR}$ recordings and until recovery. ABR equipment and software (SigGen and BioSig) from Tucker-Davis Technologies (TDT; TDT system III) were used to generate sound stimuli and acquire the auditory responses. ABR recordings were performed in a sound-proof chamber (Industrial Acoustics Co.) using broadband click stimuli (90-20 dB in $-5 \mathrm{~dB}$ steps) and tone-pips at $4,8,16,32,40,48 \mathrm{kHz}(90-10 \mathrm{~dB}$ in $-10 \mathrm{~dB}$ steps). ABR click thresholds were divided into 3 categories defined as no HL (ABR threshold between 25 and $40 \mathrm{~dB}$ ), moderate HL (ABR threshold between 45 and $55 \mathrm{~dB}$ ), and severe HL (ABR threshold between 60 and $90 \mathrm{~dB}$ ). Frequency-specific ABRs were obtained with 3 -msec tone-pips ( $0.5 \mathrm{~ms}$ rise-fall, with a $\cos 2$ envelop, at $21 / \mathrm{sec}$ tone bursts) delivered to the ear canal. Signals were detected using 3 stainless steel needle electrodes (vertex/base of skull, ipsilateral ear, low back), amplified (10,000 ×), filtered (300 Hz high-pass, $3 \mathrm{kHz}$ low-pass, $60 \mathrm{~Hz}$ notch), and averaged using alternating stimulus polarity. ABR threshold was defined as the lowest intensity at which a response was noted. The amplitude and latency of wave I were measured using BioSig software (TDT System III) and plotted against the stimulus level ( $\mathrm{dB}$ sound pressure level [SPL]). DPOAE thresholds were measured using 2 MF1 magnetic speakers connected to ER-10B microphone probe (Etymotic Research). The stimuli were applied to the ear canal in which the response was evoked using 2 primary frequencies $\mathrm{f} 1$ and $\mathrm{f} 2$ (frequency ratio $[\mathrm{f} 1 / \mathrm{f} 2]=1.2)$ and recorded at $2 \mathrm{f} 1-\mathrm{f} 2$. DPOAE responses were measured at $8,16,24$, and $32 \mathrm{kHz}$ frequencies and sound intensity from 90-20 dB at 10-dB decrements to establish threshold.

Cochlear wholemount dissection and immunofluorescence. Animals were sacrificed by $\mathrm{CO}_{2}$ overdose, perfused with PBS as described above, and cochleae were harvested. A small hole in the apex of the cochlea was created for perfusion with 4\% PFA through the oval and round window. For synaptic staining, cochleae were postfixed in $4 \%$ PFA for $2-3$ hours at $4^{\circ} \mathrm{C}$ rocking platform, decalcified with $\mathrm{RDO}$ rapid decalcifying agent (Electron Microscopy Science) for 4 minutes at room temperature, microdissected into 4 pieces (apex, mid-apex, midbase, base), and immunostained for CtBP2 and GluR2 (postsynaptic AMPA receptor subunit). Each segment was incubated in buffered serum (DPBS [Lonza], BSA [RPI], TritonX-100 [Thermo Fisher Scientific], and normal goat serum [Thermo Fisher Scientific]) for 4 hours at room temperature, followed by an overnight incubation in antibody dilution buffer (PBS, $0.1 \%$ TritonX-100, $\%$ NGS, $1 \%$ BSA) at $4^{\circ} \mathrm{C}$. Additional primary antibodies (target, species, and source) used for immunofluorescence are listed in Supplemental Table 1. Primary antibody incubations were followed by 2-hour incubation in species/isotype-matched secondary antibodies conjugated with FITC, TRITC, or Alexa Fluor at room temperature and a nuclear stain, Hoechst dye (Thermo Fisher Scientific). After immunostaining, cochlear pieces were mounted with Vectashield (Vector Laboratories) onto glass slides, coverslipped, and sealed. For HC (MyosinVIIa), SC (Sox2), and nerve fibers were stained with an antibody reactive with NF-H stainings, cochleae were postfixed in $4 \% \mathrm{PFA}$ overnight at $4^{\circ} \mathrm{C}$ on a rocking platform, but all other downstream procedures were identical to the synaptic staining. 
Cochlear midmodiolar sections. To display the structures of the SGNs and stria vascularis, cochleae were harvested, postfixed with $4 \%$ PFA overnight at $4^{\circ} \mathrm{C}$, and decalcified. Tissues were cryoprotected in $10 \%$ sucrose/PBS overnight, followed by $20 \%$ sucrose/PBS overnight at $4^{\circ} \mathrm{C}$. Cochleae were incubated in $20 \%$ sucrose/OCT (1:1) solution for 2-3 hours at room temperature, embedded in OCT medium (Electron Microscopy Sciences), and snap frozen in 2-methylbutane/dry ice. Midmodiolar sections (10$\mu \mathrm{m}$ sections) of the mouse cochleae were cut using a cryostat; sections were dried overnight at room temperature and stored in $-80^{\circ} \mathrm{C}$ until ready to stain. Frozen sections were rehydrated in PBS, and immunostaining procedures identical to the synaptic staining were performed.

Cochlear frequency mapping. After immunostaining of cochlear wholemounts, $10 \times$ confocal images of all microdissected cochlear pieces were taken in order from apex to base. Images were imported to ImageJ (Plugin/Tools/Measure Line, NIH), in which cochlear length was measured to subsequently map cochlear frequencies at $4,5.6,8,11.3,16,22.6,32,45.2$, and $64 \mathrm{kHz}$ regions for each cochlea. The frequency map was used to acquire high-resolution confocal images to compare regions sensitive to the same frequency $(21,40)$.

Confocal imaging. For cochlear regions that correspond to 8, 16, 32, and $40 \mathrm{kHz}$ frequencies, high-resolution confocal z-stack images of synapses and nerve fibers were acquired using (1.49 numerical aperture) $100 \times$ oil-immersion total internal reflection fluorescence (TIRF) objective lens through a gallium arsenide phosphide (GaAsP) detector in an Olympus FV1000 confocal microscope. Additional 1.3× or $2 \times$ digital zoom was added to the images. The $\mathrm{z}$ intervals were adjusted to span the entire synaptic region of the HCs in the z-dimension, with the z-step size of $1 \mu \mathrm{m}$ /slice. Z-stack images were collapsed into a single 2-dimensional image in Olympus Fluoview FV10 ASW 4.2 software capturing all synaptic puncta. Laser power and microscope acquisition settings were identical for all samples and regions for SGN nerve fibers, although minimal adjustments were made for image acquisition of synaptic puncta or SGN density.

Cell counting. SGNs (Tuj-1), HCs/SCs (MyosinVIIa/Sox2), and synaptic puncta (CtBP2 and GluR2) in the cochlea were manually counted with ImageJ software (Plugins $>$ Analyze $>$ Cell counter, NIH). SGN soma within Rosenthal's canal were counted from midmodiolar sections of the cochlea and the total number of soma divided by the area of Rosenthal's canal to obtain density measurements (SGN cells $/ 10,000 \mu \mathrm{m}^{2} ; n=$ 4 mice and $n=8$ cochleae). The SGN density from each of the 3 regions (apex, mid, and base) were averaged separately using measurements obtained from individual cochlea. Additionally, 40× images of cochlear wholemount were acquired, and HC and SCs were counted from cochlear segments of apex, mid, and base regions, within the span of $320 \mu \mathrm{m}$. In addition, Type II nerve fiber innervating OHCs from the 3 regions were quantified within the span of $320 \mu \mathrm{m}$ to identify the density of nerve fibers. The number of HC, SC, and nerve fibers (detected by staining for NF-H) were displayed as number of cells or fibers per $100 \mu \mathrm{m}$. Lastly, the total number of synaptic puncta (e.g., CtBP2 and GluR2 staining) was counted for 8 IHCs per image and was divided by the number of IHCs, to gain the average number of puncta associated with each IHC.

Statistics. For all experiments, $n$ refers to the number of individual mice or ears indicated in each figure legends. Results are expressed as mean or median \pm SD. Comparison between 2 groups was performed using 2-tailed unpaired $t$ test or Mann-Whitney $U$ test, depending on normal distribution of data. Categorical data was compared using Fisher's exact test. All data was analyzed for normality prior to applying parametric or nonparametric statistical analysis. For comparing more than 2 groups, $P$ values for all groups were calculated using medians by 1-way ANOVA and corrected for multiple comparisons using Kruskal-Wallis test for nonnormally distributed data and with Dunn's comparisons test. For normally distributed data, $P$ values were calculated using means by 1-way ANOVA and corrected for multiple comparisons using Tukey's post hoc test. $P$ values are indicated as ${ }^{*} P<0.05 ;{ }^{* *} P<0.01 ;{ }^{* *} P<0.001 ;{ }^{* * * *} P<0.0001$.

Study approval. All animal study procedures were approved from IACUC of the University of Alabama at Birmingham. Mice were euthanized with carbon dioxide under regulated flow. Euthanized animals were confirmed by cervical dislocation for adult mice and decapitation for mice younger than P12, as detailed in the UAB Animal Resource Program (ARP) guidelines. All animal protocols (APN9351 and APN20053) were approved from UAB IACUC to harvest tissues for this project.

\section{Author contributions}

CYWS formulated hypotheses, designed and performed the experiments, analyzed data, carried out statistical analyses, drafted figures, interpreted data, wrote the first draft, and edited the manuscript. MCS provided assistance in image processing of cochlear tissues. SP performed some repeated experiments to confirm results. $\mathrm{KH}$ provided advice on experiments and discussion on data and also edited the manuscript. 
SJ provided assistance in formulation and design of experiments to address hypotheses. WB participated in formulation of hypotheses, data interpretation, and editing and finalizing of the manuscript.

\section{Acknowledgments}

The authors would like to thank Chris Walters (Applications Engineer, TDT) for his assistance in setting up the Auditory Workstation and training for the proper use of the TDT system and software. This study was supported by the NIH grant R01 DC015980 (WB) from the National Institute of Deafness and Other Communication Disorders (NIDCD).

Address correspondence to: William J. Britt, Children's Harbor Building (CHB) 107, 1600 6th Ave. S., Birmingham, Alabama 35233, USA. Phone: 205.996.7762; Email: wbritt@peds.uab.edu.

1. Fowler KB, McCollister FP, Dahle AJ, Boppana S, Britt WJ, Pass RF. Progressive and fluctuating sensorineural hearing loss in children with asymptomatic congenital cytomegalovirus infection. J Pediatr. 1997;130(4):624-630.

2. Boppana SB, et al. Saliva polymerase-chain-reaction assay for cytomegalovirus screening in newborns. $N$ Engl J Med. 2011;364(22):2111-2118.

3. Madden C, et al. Audiometric, clinical and educational outcomes in a pediatric symptomatic congenital cytomegalovirus (CMV) population with sensorineural hearing loss. Int J Pediatr Otorhinolaryngol. 2005;69(9):1191-1198.

4. Smith RJ, Bale JF, White KR. Sensorineural hearing loss in children. Lancet. 2005;365(9462):879-890.

5. Fowler KB, Boppana SB. Congenital cytomegalovirus (CMV) infection and hearing deficit. J Clin Virol. 2006;35(2):226-231.

6. Ross SA, et al. Hearing loss in children with congenital cytomegalovirus infection born to mothers with preexisting immunity. J Pediatr. 2006;148(3):332-336.

7. Dahle AJ, Fowler KB, Wright JD, Boppana SB, Britt WJ, Pass RF. Longitudinal investigation of hearing disorders in children with congenital cytomegalovirus. J Am Acad Audiol. 2000;11(5):283-290.

8. Bernard S, Wiener-Vacher S, Van Den Abbeele T, Teissier N. Vestibular Disorders in Children With Congenital Cytomegalovirus Infection. Pediatrics. 2015;136(4):e887-e895.

9. Britt W. 2007. Virus entry into host, establishment of infection, spread in host, mechanisms of tissue damage. Human Herpesviruses: Biology, Therapy, and Immunoprophylaxis:Chapter 41.

10. Teissier N, et al. Inner ear lesions in congenital cytomegalovirus infection of human fetuses. Acta Neuropathol. 2011;122(6):763-774

11. Gabrielli L, et al. Human fetal inner ear involvement in congenital cytomegalovirus infection. Acta Neuropathol Commun. 2013;1:63.

12. Webster WS. Teratogen update: congenital rubella. Teratology. 1998;58(1):13-23.

13. Lee JY, Bowden DS. Rubella virus replication and links to teratogenicity. Clin Microbiol Rev. 2000;13(4):571-587.

14. Koontz T, et al. Altered development of the brain after focal herpesvirus infection of the central nervous system. $J$ Exp Med. 2008;205(2):423-435.

15. Rubel EW, Popper AN, Fay RR, eds. Development of the Auditory System. New York, NY: Springer; 1998.

16. $\mathrm{Ng} \mathrm{L}$, et al. Hearing loss and retarded cochlear development in mice lacking type 2 iodothyronine deiodinase. Proc Natl Acad Sci USA. 2004;101(10):3474-3479.

17. Song L, McGee JA, Walsh EJ. Consequences of combined maternal, fetal and persistent postnatal hypothyroidism on the development of auditory function in Tshrhyt mutant mice. Brain Res. 2006;1101(1):59-72.

18. Moore JK, Linthicum FH. The human auditory system: a timeline of development. Int J Audiol. 2007;46(9):460-478.

19. Bradford RD, Yoo YG, Golemac M, Pugel EP, Jonjic S, Britt WJ. Murine CMV-induced hearing loss is associated with inner ear inflammation and loss of spiral ganglia neurons. PLoS Pathog. 2015;11(4):e1004774.

20. Kosmac K, Bantug GR, Pugel EP, Cekinovic D, Jonjic S, Britt WJ. Glucocorticoid treatment of MCMV infected newborn mice attenuates CNS inflammation and limits deficits in cerebellar development. PLoS Pathog. 2013;9(3):e1003200.

21. Kujawa SG, Liberman MC. Adding insult to injury: cochlear nerve degeneration after "temporary" noise-induced hearing loss. J Neurosci. 2009;29(45):14077-14085.

22. Wan G, Gómez-Casati ME, Gigliello AR, Liberman MC, Corfas G. Neurotrophin-3 regulates ribbon synapse density in the cochlea and induces synapse regeneration after acoustic trauma. Elife. 2014;3.

23. Taberner AM, Liberman MC. Response properties of single auditory nerve fibers in the mouse. J Neurophysiol. 2005;93(1):557-569.

24. Seleme MC, Kosmac K, Jonjic S, Britt WJ. Tumor Necrosis Factor Alpha-Induced Recruitment of Inflammatory Mononuclear Cells Leads to Inflammation and Altered Brain Development in Murine Cytomegalovirus-Infected Newborn Mice. J Virol. 2017;91(8):e01983-16.

25. Driver EC, Sillers L, Coate TM, Rose MF, Kelley MW. The Atoh1-lineage gives rise to hair cells and supporting cells within the mammalian cochlea. Dev Biol. 2013;376(1):86-98.

26. Coate TM, Spita NA, Zhang KD, Isgrig KT, Kelley MW. Neuropilin-2/Semaphorin-3F-mediated repulsion promotes inner hair cell innervation by spiral ganglion neurons. Elife. 2015;4.

27. Grosse SD, Ross DS, Dollard SC. Congenital cytomegalovirus (CMV) infection as a cause of permanent bilateral hearing loss: a quantitative assessment. J Clin Virol. 2008;41(2):57-62.

28. Carraro M, Almishaal A, Hillas E, Firpo M, Park A, Harrison RV. Cytomegalovirus (CMV) Infection Causes Degeneration of Cochlear Vasculature and Hearing Loss in a Mouse Model. J Assoc Res Otolaryngol. 2017;18(2):263-273.

29. Schachtele SJ, Mutnal MB, Schleiss MR, Lokensgard JR. Cytomegalovirus-induced sensorineural hearing loss with persistent cochlear inflammation in neonatal mice. J Neurovirol. 2011;17(3):201-211.

30. Yuehua Q, et al. Inflammatory lesions of cochlea in murine cytomegalovirus-infected mice with hearing loss. Cell Biochem Biophys. 
2012;62(2):281-287.

31. Almishaal AA, et al. Natural killer cells attenuate cytomegalovirus-induced hearing loss in mice. PLoS Pathog. 2017;13(8):e1006599.

32. Perny M, Roccio M, Grandgirard D, Solyga M, Senn P, Leib SL. The Severity of Infection Determines the Localization of Damage and Extent of Sensorineural Hearing Loss in Experimental Pneumococcal Meningitis. J Neurosci. 2016;36(29):7740-7749.

33. Seal RP, et al. Sensorineural deafness and seizures in mice lacking vesicular glutamate transporter 3. Neuron. 2008;57(2):263-275

34. Trune DR, Nguyen-Huynh A. Vascular Pathophysiology in Hearing Disorders. Semin Hear. 2012;33(3):242-250.

35. Buran BN, Strenzke N, Neef A, Gundelfinger ED, Moser T, Liberman MC. Onset coding is degraded in auditory nerve fibers from mutant mice lacking synaptic ribbons. J Neurosci. 2010;30(22):7587-7597.

36. Moser T, Starr A. Auditory neuropathy--neural and synaptic mechanisms. Nat Rev Neurol. 2016;12(3):135-149.

37. Jordan S, et al. Virus progeny of murine cytomegalovirus bacterial artificial chromosome pSM3fr show reduced growth in salivary Glands due to a fixed mutation of MCK-2. J Virol. 2011;85(19):10346-10353.

38. Lutarewych MA, Quirk MR, Kringstad BA, Li W, Verfaillie CM, Jordan MC. Propagation and titration of murine cytomegalovirus in a continuous bone marrow-derived stromal cell line (M2-10B4). J Virol Methods. 1997;68(2):193-198.

39. Bantug GR, Cekinovic D, Bradford R, Koontz T, Jonjic S, Britt WJ. CD8+ T lymphocytes control murine cytomegalovirus replication in the central nervous system of newborn animals. J Immunol. 2008;181(3):2111-2123.

40. Maison S, Liberman LD, Liberman MC. Type II Cochlear Ganglion Neurons Do Not Drive the Olivocochlear Reflex: Re-Examination of the Cochlear Phenotype in Peripherin Knock-Out Mice. eNeuro. 2016;3(4):ENEURO.0207-16.2016. 